\title{
Commission 14: Atomic \& Molecular Data
}

\author{
PRESIDENT: Sveneric Johansson \\ VICE-PRESIDENT: Steven R. Federman \\ SECRETARY: Glenn M. Wahlgren \\ ORGANIZING COMMITTEE: Saul J. Adelman, Emile Biémont, \\ Steve R. Federman, Sveneric Johansson, Jim Lawler, Michael \\ Mickelson, Donald Morton, Tanya Ryabchikova, Peter L. Smith, \\ Chantal Stehle, and Glenn M. Wahlgren
}

\begin{abstract}
This triennial report from Comm. 14 covers the topics Atomic Spectra and Wavelength Standards, Atomic Transition Probabilities, Collision Processes, and Line Broadening.
\end{abstract}

Commission 14 belongs to Division XII of the IAU. The rôle of Commission 14 is to inform the astronomical community of new developments in the diverse fields of research and measurement that involve atoms, molecules and solid state particles. Conversely, it endeavors to sensitize the research communities active in those fields to the specific needs of astronomy, especially concerning basic data and modeling tools. More generally, Commission 14 tries to foster long term relations and collaborations between the two communities and, when necessary, to alert funding authorities to the specific needs of ground and space based astronomy for specific atomic and molecular data.

This report is one of the main contributions of Commission 14 to the astronomical community. It comprises Sections prepared by four of the eight Working Groups of Commission 14. It is also available on the Commission 14 web site.

\section{WORKING GROUP ON ATOMIC SPECTRA AND WAVELENGTH STANDARDS}

CHAIR: S. Johansson

CO-CHAIR: G. Nave

\subsection{Energy Level Analyses, Wavelengths and Line Classifications}

The references cited in this section are mostly papers on original laboratory research; compilations and data bases are covered in another section. The references, ordered by atomic number and spectrum, are given in parentheses following the spectral notations. They are designated by the first letter of the first author's last name and a serial number; these designations precede the full citations in the reference list at the end of the report.

H I, D I (K1), H I (K6) hfs, Li I (D2), Li I (W1) hfs, B II (M1,R1), Ne I (S4), Ne III (K5), Ca I (M4) hfs, Sc I (B2) hfs, Ti I (L3), Ti IV (R2), V I (L1) hfs, Mn I (B1, L2) hfs, Mn II (B5) hfs Cr II (S5), Ni VII (K7), Sr II (B3) hfs, Y I (N2) hfs, Nb I (B4) hfs, Mo II (N1), Cs II (S6), Sm I (O1) hfs, Sm II (M5) hfs, Bi II (D3), Pt I,II (N3, S5), Ta I (M2), Ta I (J1) hfs, Ta II (E1,M3,Z1) hfs, Ta III (A1).

The references for elements heavier than $\mathrm{Ni}(\mathrm{Z}>28)$ are limited to the first three or four spectra only, these data being of interest for solar and stellar spectroscopy. The references of the lighter elements are also incomplete, the selection being limited to those 
of highest astrophysical interest. The quality of astrophysical spectra sometimes requires that hyperfine structure (hfs) is considered in the analysis. We have therefore included some experimental data on hfs.

Current work in term analyses of high-resolution laboratory spectra (energy levels, wavelengths) of the lowest ionization stages of astrophysical significance is ongoing at Lund, Sweden (transition elements and rare earth elements), London, UK (iron group elements), NIST, USA (heavy elements, rare earths), Troitsk, Russia (heavy elements), Antigonish, Canada (higher ionization states, heavy elements) and Meudon \& Orsay, France (transition groups, theory). A number of laboratories are involved in measurements of hfs.

\subsection{Wavelength Standards}

Based on the refined energy levels of $\mathbf{H}$ \& $\mathbf{D}$ (K1) accurate wavelengths have been derived for these $\mathrm{H}$ isotopes (see Reader, 2004). Banerjee, Das, \& Natarajan (2004) present frequency measurements with an accuracy of $0.7 \mathrm{~m} \AA$ of the resonance lines of K I, which can be used to test calibration lines. Nave \& Sansonetti (2004) give reference wavelengths of Fe I, Ge I \& Pt I lines in a region around $1935 \AA$. A similar work on U I \& Th I in a limited wavelength region (6040-7550 $\AA$ ) is published by Degraffenreid \& Sansonetti (2002, D1). Accurate wavelengths for ground state lines in Ti II, Cr II, Mn II, Fe II \& Ni II have been measured in a single spectrum from a composite hollowcathode lamp (Aldenius, Johansson \& Murphy 2005). Fourier Transform Spectrometry has been used at NIST to measure calibration wavelengths of mono-isotopic mercury, ${ }^{198} \mathrm{Hg}$ (Salit et al. 2004; Veza et al. 2005)

\subsection{Larger Compilations, Reviews, Conference Proceedings}

A number of compilations on wavelengths and energy levels have been published or submitted by the spectroscopy group at NIST:

Be II (K3), Ne I (S2), Ga I-XXXI (S9), KrI-XXXVI (S3), RbI-XXXVII (S8), XeI-LIV (S1), BaI-II (C1), WI-II (K4), and special compilations of X-ray data for the Chandra Observatory: NeV-VIII (P4), MgV-X (P3), SiVI-XII (P2), S VIIXIV (P1). Ionization energies for tungsten $\left(\mathrm{W}^{2+}\right.$ to $\left.\mathrm{W}^{71+}\right)$ are compiled by Kramida \& Reader (2005). Morton (2003) has continued with a third compilation on Atomic Data for Resonance Absorption Lines, which concerns wavelengths longward of the Lyman Limit for H-Ga.

A number of papers on atomic spectroscopic data are included in proceedings of the Eigth International Colloquium on Atomic Spectra and Oscillator Strengths for Astrophysical and Laboratory Plasmas, held in Madison, WI, USA, August 2004. Invited papers are published in the Physica Scripta T Series (Lawler, Wahlgren \& Wiese 2005). A few papers on astrophysical data needs are included in the proceedings from the third and fourth international conferences, ICAMDATA, held at Gatlinburg, Tn, USA, in April 2002 (Shultz, Krstic \& Ownby 2002), and at Toki, Japan in October 2004 (Kato, Kato \& Funaba 2005), respectively. The proceedings contain review papers as well as descriptions of atomic and molecular databases. Proceedings from the EGAS (European group of Atomic Spectroscopy) conferences appear in the Physica Scripta T-series: $31^{\text {st }}$ EGAS T86 (2000) and $32^{\text {nd }}$ EGAS T95 (2001).

\subsection{Atomic Spectroscopic Data on the Internet}

A comprehensive bibliographic database on atomic spectroscopy with a search engine on various atomic parameters is available at the Institute of Spectroscopy, Troitsk, at the URL address:http://das101.isan.troitsk.ru/bibl.htm. 
The database DREAM is devoted to the rare-earth elements in the low ionization stages. It contains presently data for over 60000 transitions, many of them of astrophysical interest. More details can be found on the Web site: http://www.umh.ac.be/ astro/dream/shtml. The database has been described recently in Biémont \& Quinet (2005). A set of databases (R4) are available at NIST at following URL addresses:

http://physics.nist.gov/Handbook

This handbook by Sansonetti \& Martin (S7) includes about 10000 wavelengths for the strongest lines of all elements H-Es for neutral and singly-ionized atoms, with ionization energies, transition probabilities, line classifications, isotope information and references. URL:http://physics.nist.gov/ASD

The Atomic Spectra Database (R3) version 3.0 is fully revised since last report with new Web interface. Many new data, corrected errors, online generation of Grotrian diagrams, Saha/LTE spectra and direct access to references. Versions 2.0 and 1.3 are archived and will not be updated.

http://physics.nist.gov/Chandra.

This X-ray database for the Chandra Observatory (P5) contains Wavelengths, energy levels and transition probabilities for lines between $20 \AA$ and $170 \AA$ for $\mathrm{Ne}, \mathrm{Mg}$, Si and $\mathrm{S}$ ions

http://physics.nist.gov/IonEnergy

The database (M6) gives Ground Levels and Ionization Energies for the Neutral Atoms of 9 elements.

http://physics.nist.gov/HDEL

Theoretical values of energy levels of $H$ and D for quantum numbers $n=1$ to 200 .

An Atomic Energy Levels and Spectra Bibliographic Database is in preparation. It contains about 8500 references between July 1968-December 2000 on atomic energy levels and spectra. It will be merged with the transition probability and line broadening bibliographic databases.

\section{References}

Aldenius, M., Johansson, S. \& Murphy, M. 2006, Mon.Not.R.Astr.Soc. in press

A1 Azarov, V. I., Tchang-Brillet, W.-Ü. L., Wyart, J.-F. \& Meijer, F. G. 2003, Physica Scripta 67,190

Banerjee, A., Das, D. \& Natarajan, V. 2004 J.Opt.Soc.Am.B 21, 79

Biémont, E. \& Quinet, P. 2005, J. Electr. Spectr. Rel. Phen. 144-147, 23-25

B3 Barwood, G.P., Gao, K., Gill, P., Huang, G. \& Klein, H.A. 2003 Phys.Rev.A 67, 013402

B2 Basar, G., Basar, G., Acar, F.G., Ozturk, I.K. \& Kroger, S. 2004 Physica Scripta 69, 189

B1 Blackwell-Whitehead, R.J., Pickering, J.C., Pearse, O. \& Nave, G. 2005 Ap. J. Suppl. 157, 402

B5 Blackwell-Whitehead, R.J., Toner, A., Hibbert, A., Webb, J. \& Ivarsson, S. 2005 Mon.Not.R.Astron.Soc. 364, 705

B4 Bouzed, A., Kröger, S., Zimmerman, D., Kronfeldt, H.-D. \& Guthöhrlein, G.H., 2003 Eur.J.Phys.D 23, 57

C1 Curry, J.J. 2004 J.Phys.Chem.Ref.Data 33, 725

D1 DeGraffenreid, W. \& Sansonetti, C.J. 2002 J.Opt.Soc.Am.B 19, 1711

D2 DeGraffenreid, W. \& Sansonetti, C.J. 2003 Phys.Rev.A 67, 012509

D3 Dolk, L., Litzén, U. \& Wahlgren, G.M. 2002 Astron. Astrophys. 388, 692

E1 Eriksson, M., Litzén, U., Wahlgren, G.M. \& Leckrone, D.S. 2002 Physica Scripta 65, 480

J1 Jaritz, N., Guthöhrlein, G.H., Windholz, L., Messnarz, D., Engleman, R., Pickering, J.C. \& Jäger, H. 2004, Physica Scripta 69, 441

Kato, T., Kato, D. \& Funaba, H. 2005 AIP Conference Proceedings 771 
K6 Kolachevsky, N., Fischer, M., Karshenboim, S.G. \& Hänsch, T. 2004 Phys.Rev.Lett. 92, 033003

K1 Kotochigova, S., Mohr, P.J. \& Taylor, B.N. 2002 Can.J.Phys. 80, 1373

K2 Kotochigova, S., Mohr, P.J. \& Taylor B.N. 2004 The Energy Levels of Hydrogen and Deuterium (NIST, Gaithersburg, MD, USA)

K3 Kramida, A.E. 2005 Physica Scripta, 72, 309

K4 Kramida, A.E. 2006 J.Phys.Chem.Ref.Data, in press

K5 Kramida, A.E. \& Nave, G. 2006 Eur.J.Phys.D, 37, 1

Kramida, A.E. \& Reader, J. 2006 At.Data Nucl.Data tables, in press

K7 Kildiyarova R.R., Churilov S.S., Joshi Y.N. \& Ryabtsev A.N. 2005 J.Opt.Soc.Am.B 22, 884

Lawler, J. E., Wahlgren, G. M. \& Wiese, W. L.: editors, Physica Scripta T119 (2005)

L1 Lefèbvre, P.-H., Garnir, H.-P. \& Biémont, E. 2002 Physica Scripta 66, 363

L2 Lefèbvre, P.-H., Garnir, H.-P. \& Biémont, E. 2003 Astron.Astrophys. 404, 1153

L3 Litzén, U. 2005 Physica Scripta, 71, 471

M5 Masterman, D., Rosner, S.D., Scholl, T.J., Sharikova, A. \& Holt, R.A. 2003 Can.J.Phys. 81, 1389

M1 Martinson I., Awaya Y., Ekberg J.O., Kink I., Mannervik S. \& Ryabtsev A.N. 2003 J.Phys.B 36,419

M2 Messnarz, D., Jaritz, N., Arcimowics, B.,Zilio, V.O., Engleman, R., Pickering, J.C., Jäger, H., Guthöhrlein, G.H. \& Windholz, L. 2003 Physica Scripta 68, 170

M3 Messnarz, D. \& Guthöhrlein, G.H. 2003 Physica Scripta 67, 59

M6 Martin, W.C. et al. 2003 Ground Levels and Ionization Energies for the Neutral Atoms (NIST, Gaithersburg, MD, USA)

M4 Mortensen, A., Lindballe, J.J.T., Jense, I.S., Staanum, P. \& Voigt, D. 2004 Phys.Rev.A 69, 042502

Morton, D.C. 2003 ApJ. Suppl 149, 205

N3 Nave, G. \& Sansonetti, C.J. 2004 J.Opt.Soc.Am.B 21, 442

N1 Nilsson, H. \& Pickering, J. C. 2003 Physica Scripta 67, 223

N2 Nakada, H., Jin, W.G., Minowa, T., Katsuragawa, H. \& Uematsu, H. 2004 Jap.J.Appl.Phys 43, A6423

O1 Oh, M.K., Choi, W, Jeon, J.H., Lee, M., Choi, Y., Park, S., Lee, J.H. \& An, K. 2004 Spectrochim. Acta B 59, 1919

P1 Podobedova, L.I., Musgrove, A., Kelleher, D.E.,Reader, J. \& Wiese, W.L. 2003 J.Phys.Chem.Ref.Data 32, 1367

P2 Podobedova, L.I., Kelleher, D.E., Reader, J. \& Wiese, W.L. 2004 J.Phys.Chem.Ref.Data 33, 471

P3 Podobedova, L.I., Kelleher, D.E., Reader, J. \& Wiese, W.L. 2004 J.Phys.Chem.Ref.Data 33, 495

P4 Podobedova, L.I., Kelleher, D.E., Reader, J. \& Wiese, W.L. 2004 J.Phys.Chem.Ref.Data 35, 525

Podobedova, L., Musgrove, A., Kelleher, D., Reader, J \& Wiese, W. 2003, Spectral Data for the Chandra X-ray Observatory, Version 1.0 (NIST, Gaithersburg, MD, USA).

Reader, J. 2004 Appl. Spectrosc. 58, 12

R1 Ryabtsev A.N., Kink I., Awaya Y., Ekberg J.O., Mannervik S., Ölme A. \& Martinson I. 2005 Physica Scripta 71, 489

R2 Ryabtsev A.N., Churilov S.S. \& Kononov E.Ya. 2005 Optics \&3 Spectr. 98, 519

R3 Ralchenko, Yu, Jou, F.-C., Kelleher, D.E., Kramida, A.E., Musgrove, A., Reader, J., Wiese, W.L. \& Olsen, K. 2005 Atomic Spectra Database Version 3.0.2 (NIST, Gaithersburg, MD, USA)

R4 Ralchenko, et al. 2006 J. Plasma E Fusion Research, in press.

Salit, M.L., Sansonetti, C. J., Veza, D \& Travis, J.C. 2004 J.Opt.Soc.Am.B 21, 1543

S1 Saloman, E.B. 2004 J.Phys.Chem.Ref.Data 33, 765

S2 Saloman, E.B. \& Sansonetti, C.J. 2004 J.Phys.Chem.Ref.Data 33, 1113

S3 Saloman, E.B. 2005 J.Phys.Chem.Ref.Data, submitted

S4 Sansonetti, C.J., Blackwell, M.M. \& Salomon, E.B. 2004 J.Res.NIST 109, 371 
S5 Sansonetti, C.J., Kerber, F., Reader, J. \& Rosa, M.R. 2004 Ap.J.Suppl.Ser. 153, 555

S6 Sansonetti, C.J. \& Andrew, K.L. 2005 Physica Scripta 71, 362

S7 Sansonetti, J.E. \& Martin, W.C. 2005 Phys. Chem. Ref. Data, 34, 1559

S8 Sansonetti, J.E. 2006 Phys.Chem.Ref.Data, in press

Shultz, D.R., Krstić, P. S. \& Ownby, F. 2002 AIP Conference Proceedings 636

S9 Shirai, T., Reader, J., Kramida, A.E. \& Sugar, J. 2006, J.Phys.Chem.Ref.Data, submitted

W1 Walls, J., Ashby, R., Clarke, J.J., Lu, B. \& Van Wijngaerden, W.A. 2003 Eur.Phys.J.D 22, 159

Veza, D, Salit, M.L., Sansonetti, C. J. \& Travis, J.C. 2006 J.Phys.B, 38, 3739

Z1 Zilio, V. \& Pickering, J.C. 2002 Mon.Not.R.Astr.Soc. 334, 48

\section{WORKING GROUP ON ATOMIC TRANSITION PROBABILITIES}

CHAIR: G. M. Wahlgren

CO-CHAIR: J. R. Fuhr

Members of the astronomy and physics communities continue to produce new data on transition probabilities and oscillator strengths that are crucial to spectral analysis of astronomical objects. The techniques include laboratory experiment, ab initio calculations and direct inferrence from stellar spectra. In addition to creating new data, progress continues in providing easily accessible data sets.

The attached bibliography for the period 2002-2005 consists of 182 references. Of these, 145 publications involve quantum mechanical calculations, many as the sole technique. The reference list of ions is represented by 96 elements and a wide range of ionic states. The distribution among ions states is not equitable, but reflects two groupings: the lowest three ions and the highest ionization states.

The distribution by spectrum also reflects the techniques employed. Studies of highionization states are invariably of a theoretical nature, while those for the lowest ionization states include all of the experimental analyses reported. Among the experimental works, the techniques involve study of emission lines and the recording of atomic lifetimes, usually by the method of laser-induced fluorescence. The maturity of this technique is now providing benefits to many applications. The application of absorption spectroscopy appears currently to be a rare occurrence.

It is also apparent that work on atomic data is generally motivated by the needs of astronomical-based projects, and not to enhance the overall knowledge of atomic parameters. Of continued interest have been elements from the iron group, the lanthanides, and even heavier stable elements. The experimental work conducted in support of these programs is limited to the neutral and two lowest ions by available instrumentation and light sources, often being hollow-cathode designs.

The following major compilations of transition probability data have been published during the latest 3-year period:

(a) The MCHF/MCDHF Collection on the Web has been established by C. Froese Fischer et al.: http://www.vuse.vanderbilt.edu/ cff/mchf_collection/. It contains a large number of transitions for Li-like through Ar-like ions of various chemical elements, 
principally the lighter elements through atomic number $\mathrm{Z}=30$. The data include various types of electric-dipole forbidden lines and are obtained from sophisticated multiconfiguration Hartree Fock (MCHF) or, for the heavier, more highly ionized species, from multi-configuration Dirac Hartree Fock (MCDHF) calculations. The tabulations provide data for fine-structure transitions and include other important spectroscopic data for each line, and are structured similar to the NIST tables. Another large compilation was recently published: Breit-Pauli energy levels, lifetimes, and transition probabilities for the beryllium-like to neon-like sequences, C. F. Fischer and G. Tachiev, At. Data Nucl. Data Tables 87, 1-184 (2004).

(b) Transition probabilities for the resonance lines of selected elements have been compiled by Morton: Atomic data for resonance absorption lines. III. Wavelengths longward of the Lyman limit for the elements hydrogen to gallium, D. C. Morton, Astrophys. J., Suppl. Ser. 149, 205-238 (2003); Erratum: 151, 403 (2004).

(c) NIST Atomic Transition Probabilities, J. R. Fuhr and W. L. Wiese, in CRC Handbook of Chemistry and Physics, 86th Edition, 10-93-10-155 (Edited by D. R. Lide, CRC Press, Boca Raton, FL, 2005)

The use of electronic databases continues to grow as more data and bibliographies are made available in this medium. Several of the most useful databases active during this reporting period include:

(a) Version 7.0 of the NIST Atomic Transition Probability Bibliographic Database now contains 6570 references, dating from 1914 through June 2002. For this version, 454 bibliographic entries have been added.

(b) The availability of transition probability and oscillator strength data computed under the TOPbase and Opacity Projects is accessible at http://vizier.u-strasbg.fr/topbase/ topbase.html for astrophysically abundant ions $(Z \leqslant 26)$. The OPserver at this site allows opacity calculations for arbitrary chemical mixtures.

(c) The D.R.E.A.M. database (Database on Rare Earths at Mons University) continued to have data added for wavelengths, energy levels, oscillator strengths and radiative lifetimes for neutral, singly and doubly-ionized rare earth elements. These data reflect input from calculations, lifetime measurements, and experimental branching fractions.

Discussions around the availability of data, along with the presentation of techniques, continued through the proceedings of the 8th International Colloquium on Atomic Spectra and Oscillator Strengths (ASOS8) [105]. Several review articles present techniques in determining experimental oscillator strengths, including the laser-induced fluorescence, electron-beam ion-traps, and storage rings.

The main body of this report is a bibliography of selected recent literature references, which contain new transition probability data of astrophysical interest produced during the last three-year period. Thus, this new selected bibliography continues where the last working group left off. As in the previous reports, the bibliographical material is ordered with respect to element and stage of ionization. One table provides an overview of the bibliographical data by spectrum. We were selective in our choice of these references, i.e., all papers had to contain a significant amount of numerical data, normally for more than 
ten spectral lines. The references are identified by a running number, which refers to the general reference list given in this report. In the general reference list, the literature is ordered alphabetically according to the first author, and each reference contains one or more code letters indicating the method applied by the authors. These code letters are defined as follows:

\section{THEORETICAL METHODS:}

Q quantum mechanical calculations

\section{EXPERIMENTAL METHODS:}

A measurements in absorption (absorption tube, etc.)

E measurements in emission (arc, hollow cathode, etc.)

L lifetime measurements (laser-induced fluorescence, beam-laser and beam-foil spectroscopy, etc.)

M miscellaneous experimental methods (for example, Stark effect, astrophysical measurements, etc.)

\section{OTHER:}

CP data compilations

$\sim \mathrm{CM}$ comments

$\sim \mathrm{F}$ forbidden transitions (not electric dipole)

$\sim \mathrm{R}$ relative values only

Table 1. Important Literature References

Ac LXXVII: 139

Ac LXXXII: 174

Ag XXXV: 139

Ag XL: 174

Al I: 18

Al II: 177

Al VI: 99

Am LXXXIII: 139

Ar I: 14,85

Ar V: 157

Ar VI: 77, 139

Ar VII: 67

Ar XI: 99, 115, 121, 174

Ar XII: 66, 115, 121

Ar XIII: 1, 106, 115, 121

Ar XIV: 1, 115, 121
Ar XV: 115, 121

Ar XVI: 112, 115, 121

Ar XVII: 115, 121, 140

As XXI: 139

As XXVI: 174

At LXXIII: 139

At LXXVIII: 174

Au LXVII: 139

Au LXXII: 174

B II: 74

Ba I: 52

Ba II: 52

Ba XLIV: 139

Ba XLIX: 174
Bi LXXI: 139

Bi LXXVI: 174

Bk LXXXV: 139

Br XXIII: 139

Br XXVIII: 174

C I: 44, 73, 167, 168, 176

C II: 50

C III: 74

C IV: 6

C V: 140

Ca I: 18, 44, 68

Ca VII: 102

Ca VIII: 101, 139

Ca IX: 94, 150

Ca XIII: 99, 174

Ca XIX: 95 
Table 1. Important Literature References (cont.)

Cd I: 160

Cd II: 111, 160

Cd XXXVI: 139

Cd XLI: 174

Ce I: 163

Ce II: 163

Ce III: 35

Ce XLVI: 139

Ce LI: 174

Cf LXXXVI: 139

Cl I: 19

Cl II: 19, 58, 141, 147, 149

Cl III: 19, 141

Cl IV: 19

Cl V: 19, 47, 139

Cl VI: 19, 67, 123

Cl VII: 19

Cl VIII: 19

Cl IX: 19

Cl X: 19, 41, 99, 174

Cl XI: 19

Cl XII: 19

Cl XIII: 19

Cl XIV: 19

Cl XV: 19

Cl XVI: 19

Cm LXXXIV: 139

Co XV: 139

Co XVII: 136, 137

Co XX: 99, 174

Cr I: 18,44

Cr II: 18

Cr VIII: 114

Cr XII: 139

Cr XVII: 99, 174

Cr XIX: 7

Cs XLIII: 139

Cs XLVIII: 174

Cu II: 65

Cu XIV: 152
Cu XVII: 139

Cu XXII: 99, 174

Dy III: 175

Dy LIV: 139

Dy LIX: 174

Er I: 164

Er II: 158

Er LVI: 139

Er LXI: 174

Es LXXXVII: 139

Eu III: 133

Eu LI: 139

Eu LVI: 174

Fe I: 44

Fe II: $13,49,82,89,126,128,138,142$

Fe III: 126

Fe IV: 70, 118, 126

Fe V: 126

Fe VI: 126

Fe VII: 126, 170

Fe VIII: 126, 171, 172

Fe IX: 126, 171

Fe X: 2, 59, 153

Fe XI: 8, 27, 153

Fe XII: 38, 153

Fe XIII: 4, 153, 156, 157, 166

Fe XIV: 15, 75, 139

Fe XV: 96

Fe XVI: 96, 135, 137

Fe XVII: 3, 9, 24, 120, 181

Fe XVIII: 92

Fe XIX: 91, 99, 174

Fe XX: 90, 119, 127

Fe XXI: 127

Fe XXII: 127

Fe XXIII: 127

Fe XXIV: 71, 112, 127

Fe XXV: 127

Fm LXXXVIII: 139

Fr LXXV: 139

Fr LXXX: 174
Ga XIX: 139

Ga XXIV: 174

Gd I: 159

Gd II: 159

Gd III: 34

Gd LII: 139

Gd LVII: 174

Ge XX: 139

Ge XXV: 174

Hf LX: 139

Hf LXV: 174

Hg LXVIII: 139

Hg LXXIII: 174

Ho I: 122

Ho II: 104

Ho LV: 139

Ho LX: 174

I XLI: 139

I XLVI: 174

In II: 125

In XXXVII: 139

In XLII: 174

Ir I: 87

Ir II: 86

Ir LXV: 139

Ir LXX: 174

K I: 146

K VII: 139

K VIII: 37,56

K XII: 99, 174

K XIV: 7

Kr I: 14

Kr XXIII: 157

Kr XXIV: 139

Kr XXIX: 174

La I: 31

La II: 62,138 
Table 1. Important Literature References (cont.)

\begin{tabular}{|c|c|c|}
\hline \multirow{3}{*}{$\begin{array}{l}\text { La XLV: } 139 \\
\text { La L: } 174\end{array}$} & Ne V: 129 & Pm LIV: 174 \\
\hline & Ne VI: 129 & \\
\hline & Ne VII: 129,173 & Po LXXII: 139 \\
\hline Lu I: 53 & Ne VIII: 129 & Po LXXVII: 174 \\
\hline Lu LIX: 139 & Ne IX: 140 & \\
\hline \multirow[t]{2}{*}{ Lu LXIV: 174} & & Pr I: 30 \\
\hline & Ni I: 18,88 & Pr II: 33, 144 \\
\hline Mg I: 18 & Ni IV: 113 & Pr XLVII: 139 \\
\hline Mg II: 46, 108, 109 & Ni XII: 145 & Pr LII: 174 \\
\hline $\mathrm{Mg} \mathrm{V}: 99,130$ & Ni XIII: 10,152 & \\
\hline Mg VI: 130 & Ni XIV: 10 & Pt I: 60 \\
\hline Mg VII: 130 & Ni XV: 10 & Pt LXVI: 139 \\
\hline Mg VIII: 130 & Ni XVI: 10, 75, 139 & Pt LXXI: 174 \\
\hline Mg IX: 130 & Ni XVII: 57 & \\
\hline Mg X: 130 & Ni XVIII: 134,137 & Pu LXXXII: 139 \\
\hline \multirow[t]{2}{*}{ Mg XI: 165} & Ni XXI: $26,99,174$ & \\
\hline & Ni XXV: 154 & Ra LXXVI: 139 \\
\hline Mn I: 39 & & Ra LXXXI: 174 \\
\hline Mn II: 151 & Np LXXXI: 139 & \\
\hline Mn XIII: 139 & & Rb XXV: 139 \\
\hline Mn XVIII: 99, 174 & O I: $73,176,180$ & Rb XXX: 174 \\
\hline \multirow[t]{2}{*}{ Mn XX: 7} & O II: 180 & \\
\hline & O III: 180 & Re LXIII: 139 \\
\hline Mo II: 124 & O IV: $50,97,116,169,180$ & Re LXVIII: 174 \\
\hline Mo XXX: 139 & $\mathrm{O} V: 16,117,180$ & \\
\hline \multirow[t]{2}{*}{ Mo XXXV: 174} & O VI: 5,180 & Rh XXXIII: 139 \\
\hline & O VII: 140 & Rh XXXVIII: 174 \\
\hline \multicolumn{3}{|l|}{ N I: 179} \\
\hline N III: 50,64 & Os I: 87 & Rn I: 14 \\
\hline N IV: 16,64 & Os II: 86 & Rn LXXIV: 139 \\
\hline N V: 64 & Os LXIV: 139 & Rn LXXIX: 174 \\
\hline \multirow[t]{2}{*}{ N VI: 140} & Os LXIX: 174 & \\
\hline & & Ru XXXII: 139 \\
\hline Na I: 146 & P II: 148 & Ru XXXVII: 174 \\
\hline Na III: 83 & P III: 78, 139 & \\
\hline \multirow[t]{2}{*}{ Na IV: 99} & P IV: 67,79 & S II: 84 \\
\hline & P VIII: 99, 174 & S IV: 139 \\
\hline Nb XXIX: 139 & & S V: 67 \\
\hline \multirow[t]{2}{*}{ Nb XXXIV: 174} & Pa LXXIX: 139 & S VII: 42 \\
\hline & Pa LXXXIV: 174 & S VIII: 132 \\
\hline Nd I: 30 & & S IX: $23,43,99,132,174$ \\
\hline Nd II: $61,143,161$ & Pb LXX: 139 & S X: $21,43,93,132$ \\
\hline Nd XLVIII: 139 & Pb LXXV: 174 & S XI: 103,132 \\
\hline \multirow[t]{2}{*}{ Nd LIII: 174} & & S XII: 132 \\
\hline & Pd XXXIV: 139 & S XIII: 132 \\
\hline Ne I: 14 & Pd XXXIX: 174 & S XIV: 132 \\
\hline Ne II: 178 & & S XV: 95 \\
\hline Ne III: 25, 100 & Pm XLIX: 139 & \\
\hline
\end{tabular}


Table 1. Important Literature References (cont.)

Sb XXXIX: 139

Sb XLIV: 174

Sc IX: 139

Sc XIV: 99, 174

Sc XVI: 7

Se XXII: 139

Se XXVII: 174

Si I: $18,44,51,69$

Si II: 45

Si III: 63

Si V: 17

Si VI: 131

Si VII: 20, 99, 131, 174

Si VIII: 22, 131

Si IX: 131

Si X: 131, 155

Si XI: 131

Si XII: 131

Si XIII: 140

Sm II: 143, 162

Sm III: 32

Sm L: 139

Sm LV: 174

Sn II: 11, 48

Sn XXXVIII: 139

Sn XLIII: 174

Sr XXVI: 139
Sr XXXI: 174

Ta III: 12

Ta LXI: 139

Ta LXVI: 174

Tb LIII: 139

Tb LVIII: 174

Tc XXXI: 139

Tc XXXVI: 174

Te XL: 139

Te XLV: 174
V XI: 139

V XVI: 99, 174

V XVIII: 7

W LXII: 139

W LXVII: 174

Xe I: $14,54,55$

Xe II: 182

Xe V: 28

Xe VI: 29

Xe VIII: 72

Xe XLII: 139

Xe XLVII: 174

Th LXXVIII: 139 Y XXVII: 139

Th LXXXIII: 174 Y XXXII: 174

Ti I: 44

Yb I: 107

Ti II: 81

Yb II: 36

Ti X: 76, 139

Yb LVIII: 139

Ti XI: 56

Yb LXIII: 174

Ti XV: 99, 174

Ti XVII: $7 \quad$ Zn I: $18,40,98$

Zn II: 40,80

Tl LXIX: 139

Zn XVIII: 139

Tl LXXIV: 174

Zn XXIII: 99, 174

Zr III: 110

Tm LVII: 139

Zr XXVIII: 139

Tm LXII: 174

Zr XXXIII: 174

U LXXX: 139

U LXXXV: 174

\section{References}

1. Aggarwal, K. M., Keenan, F. P., Nakazaki, S., A\&A 436, 1141-1150 (2005) Q, QF

2. Aggarwal, K. M., Keenan, F. P., A\&A 427, 763-767 (2004) Q, QF

3. Aggarwal, K. M., Keenan, F. P., Kisielius, R., A\&A 420, 783-788 (2004) Q, QF

4. Aggarwal, K. M., Keenan, F. P., A\&A 418, 371-385 (2004) Q

5. Aggarwal, K. M., Keenan, F. P., Phys. Scr. 70, 222-234 (2004) Q

6. Aggarwal, K. M., Keenan, F. P., Phys. Scr. 69, 385-397 (2004) Q

7. Aggarwal, K. M., Keenan, F. P., Msezane, A. Z., A\&A 401, 377-383 (2003) Q

8. Aggarwal, K. M., Keenan, F. P., MNRAS 338, 412-424 (2003) Q

9. Aggarwal, K. M., Keenan, F. P., Msezane, A. Z., ApJS 144, 169-210 (2003) Q

10. Aggarwal, K. M., Keenan, F. P., Msezane, A. Z., At. Data Nucl. Data Tables 85, 453-494 (2003) Q

11. Alonso-Medina, A., Colón, C., Rivero, C., Phys. Scr. 71, 154-158 (2005) Q 
12. Azarov, V. I., Tchang-Brillet, W.-Ü. L., Wyart, J.-F., Meijer, F. G., Phys. Scr. 67, 190-207 (2003) Q

13. Beck, D. R., Phys. Scr. 71, 447-452 (2005) Q

14. Beck, D. R., Phys. Rev. A 66, 034502 (2002) QF

15. Beiersdorfer, P., Träbert, E., Pinnington, E. H., ApJ 587, 836-840 (2003) LF

16. Bell, K. L., Phys. Scr. T100, 64 (2002) Q

17. Ben Nessib, N., Elabidi, H., Cornille, M., Dubau, J., Phys. Scr. 72, 23-30 (2005) Q

18. Bensby, T., Feltzing, S., Lundstrm, I., A\&A 410, 527-551 (2003) M

19. Berrington, K. A., Nakazaki, S., At. Data Nucl. Data Tables 82, 1 (2002) Q

20. Bhatia, A. K., Landi, E., ApJ 585, 587-597 (2003) Q, QF

21. Bhatia, A. K., Landi, E., ApJS 147, 409-419 (2003) Q, QF

22. Bhatia, A. K., Landi, E., At. Data Nucl. Data Tables 85, 317-376 (2003) Q, QF

23. Bhatia, A. K., Landi, E., At. Data Nucl. Data Tables 85, 169-253 (2003) Q, QF

24. Bhatia, A. K., Doschek, G. A., At. Data Nucl. Data Tables 85, 1-45 (2003) Q, QF

25. Bhatia, A. K., Thomas, R. J., Landi, E., At. Data Nucl. Data Tables 83, 113-152 (2003) Q, QF

26. Bhatia, A. K., Landi, E., Mason, H. E., At. Data Nucl. Data Tables 83, 71-112 (2003) Q, QF

27. Bhatia, A. K., Doschek, G. A., Eissner, W., At. Data Nucl. Data Tables 82, 211-255 (2002) Q, QF

28. Biémont, E., Quinet, P., Zeippen, C. J., Phys. Scr. 71, 163-169 (2005) Q

29. Biémont, E., Buchard, V., Garnir, H.-P., Lefébvre, P.-H., Quinet, P., Eur. Phys. J. D 33, 181-191 (2005) L, Q

30. Biémont, E., Quinet, P., Svanberg, S., Xu, H. L., J. Phys. B 37, 1381-1389 (2004) L

31. Biémont, E., Quinet, P., Svanberg, S., Xu, H. L., Eur. Phys. J. D 30, 157-162 (2004) L, Q

32. Biémont, E., Garnir, H. P., Litzén, U., Nielsen, K., Quinet, P., Svanberg, S., Wahlgren, G. M., Zhang, Z. G., A\&A 399, 343-349 (2003) E, L, Q

33. Biémont, E., Lefèbvre, P.-H., Quinet, P., Svanberg, S., Xu, H. L., Eur. Phys. J. D 27, 33-41 (2003) L, Q

34. Biémont, E., Kohnen, G., Quinet, P., A\&A 393, 717-720 (2002) Q

35. Biémont, E., Quinet, P., Ryabchikova, T. A., MNRAS 336, 1155-1160 (2002) Q

36. Biémont, E., Quinet, P., Dai, Z., Zhankui, J., Zhiguo, Z., Xu, H., Svanberg, S., J. Phys. B 35, 4743-4749 (2002) L, Q

37. Biémont, E., Dumont, P.-D., Garnir, H. P., Palmeri, P., Quinet, P., Eur. Phys. J. D 20, 199-204 (2002) Q

38. Biémont, E., Palmeri, P., Quinet, P., Träbert, E., Zeippen, C. J., Eur. Phys. J. D 20, 37-44 (2002) Q, QF

39. Blackwell-Whitehead, R. J., Xu, H. L., Pickering, J. C., Nave, G., Lundberg, H., MNRAS 361, 1281-1286 (2005) E, L

40. Blagoev, K. B., Malcheva, G., Penchev, V., Biémont, E., Xu, H. L., Persson, A., Svanberg, S., Phys. Scr. 69, 433-440 (2004) L, Q

41. Bogdanovich, P., Karpuškienè, R., Martinson, I., Phys. Scr. 67, 44-51 (2003) Q

42. Borges, F. O., Cavalcanti, G. H., Trigueiros, A. G., Jupén, C., JQSRT 83, 751-763 (2004) Q

43. Borges, F. O., Cavalcanti, G. H., Trigueiros, A. G., JQSRT 78, 119-129 (2003) Q

44. Borrero, J. M., Bellot Rubio, L. R., Barklem, P. S., del Toro Iniesta, J. C., A\&A 404, 749-762 (2003) M 
45. Cavalcanti, G. H., Borges, F. O., Mania, A. J., Orloski, R. V., Trigueiros, A. G., JQSRT 90, 291-308 (2005) Q

46. Charro, E., Martín, I., ApJ 585, 1191-1196 (2003) QF

47. Choudhury, K. B., Deb, N. C., Roy, K., Msezane, A. Z., Eur. Phys. J. D 27, 103-107 (2003) Q

48. Colón, C., Alonso-Medina, A., A\&A 422, 1109-1111 (2004) Q

49. Corrégé, G., Hibbert, A., ApJ 627, L157-L159 (2005) Q

50. Corrégé, G., Hibbert, A., At. Data Nucl. Data Tables 86, 19-34 (2004) Q

51. Coutinho, L. H., Trigueiros, A. G., JQSRT 75, 357 (2002) Q

52. Curry, J. J., J. Phys. Chem. Ref. Data 33, 725-746 (2004) CP

53. Dai, Z., Zhankui, J., Xu, H., Zhiguo, Z., Svanberg, S., Bimont, E., Lefèbvre, P. H., Quinet, P., J. Phys. B 36, 479-487 (2003) L, Q

54. Das, M. B., Karmakar, S., Phys. Scr. 71, 170-171 (2005) L

55. Das, M. B., Karmakar, S., Eur. Phys. J. D 32, 285-288 (2005) L

56. Das, R., Deb, N. C., Roy, K., Msezane, A. Z., A\&A 416, 375-381 (2004) Q

57. Das, R., Deb, N. C., Roy, K., Msezane, A. Z., Phys. Scr. 67, 401-406 (2003) Q

58. Deb, N. C., Crothers, D. S. F., Felfli, Z., Msezane, A. Z., J. Phys. B 36, p. L47-L55 (2003) Q

59. Del Zanna, G., Berrington, K. A., Mason, H. E., A\&A 422, 731-749 (2004) CM, CMF, Q, QF

60. Den Hartog, E. A., Herd, M. T., Lawler, J. E., Sneden, C., Cowan, J. J., Beers, T. C., ApJ 619, 639-655 (2005) E, L

61. Den Hartog, E. A., Lawler, J. E., Sneden, C., Cowan, J. J., ApJS 148, 543-566 (2003) E, L

62. Derkatch, A., Ilyinsky, L., Mannervik, S., Norlin, L.-O., Rostohar, D., Royen, P., Schef, P., Biémont, E., Phys. Rev. A 65, 062508 (2002) LF, QF

63. Djeniže, S., Dimitrijevič, M. S., Srečkovič, A., Bukvič, S., A\&A 396, 331-336 (2002) ER, Q

64. Djeniže, S., Srećković, A., Bukvić, S., Eur. Phys. J. D 20, 11-16 (2002) E

65. Dong, C. Z., Fritzsche, S., Phys. Rev. A 72, 012507 (2005) Q

66. Eissner, W., Landi, E., Bhatia, A. K., At. Data Nucl. Data Tables 89, 139-194 (2005) Q, QF

67. Fan, J., Zheng, N. W., Chem. Phys. Lett. 400, 273-278 (2004) Q

68. Fischer, C. F., Tachiev, G., Phys. Rev. A 68, 012507 (2003) Q

69. Froese Fischer, C., Phys. Rev. A 71, 042506 (2005) Q, QF

70. Froese Fischer, C., Rubin, R. H., MNRAS 355, 461-474 (2004); Erratum: 355, 1400 (2004) QF

71. Galavís, M. E., Bautista, M. A., Mendoza, C., Rev. Mex. Fis. 49(3), 63-66 (2003) (Span.) Q

72. Gallardo, M., Raineri, M., Giuliani, M., Lagorio, C., Padilla, S., Sarmiento, R., Reyna Almandos, J. G., JQSRT 95, 365-372 (2005) Q

73. Golly, A., Jazgara, A., Wujec, T., Phys. Scr. 67, 485-490 (2003) E

74. Gou, B.-C., Wang, F., Phys. Rev. A 69, 042513 (2004) Q

75. Gupta, G. P., Msezane, A. Z., At. Data Nucl. Data Tables 89, 1-44 (2005) Q

76. Gupta, G. P., Msezane, A. Z., Phys. Scr. 70, 235-240 (2004) Q

77. Gupta, G. P., Msezane, A. Z., Phys. Scr. 69, 273-282 (2004) Q

78. Gupta, G. P., Msezane, A. Z., Phys. Scr. 66, 354-362 (2002) Q

79. Gupta, G. P., Msezane, A. Z., Phys. Scr. 66, 43-50 (2002) Q

80. Harrison, S. A., Hibbert, A., MNRAS 340, 1279-1286 (2003) Q 
81. Hartman, H., Schef, P., Lundin, P., Ellmann, A., Johansson, S., Lundberg, H., Mannervik, S., Norlin, L.-O., Rostohar, D., Royen, P., MNRAS 361, 206-210 (2005) LF, $\mathrm{MF}$

82. Hartman, H., Derkatch, A., Donnelly, M. P., Gull, T., Hibbert, A., Johansson, S., Lundberg, H., Mannervik, S., Norlin, L.-O., Rostohar, D., Royen, P., Schef, P., A\&A 397, 1143-1149 (2003) MF, QF

83. Hibbert, A., J. Phys. B 36, 4703-4715 (2003) CM, Q

84. Irimia, A., Froese Fischer, C., Phys. Scr. 71, 172-184 (2005) Q, QF

85. Irimia, A., Fischer, C. F., J. Phys. B 37, 1659-1672 (2004) Q

86. Ivarsson, S., Wahlgren, G. M., Dai, Z., Lundberg, H., Leckrone, D. S., A\&A 425, 353-360 (2004) E, L

87. Ivarsson, S., Andersen, J., Nordström, B., Dai, X., Johansson, S., Lundberg, H., Nilsson, H., Hill, V., Lundqvist, M., Wyart, J. F., A\&A 409, 1141-1149 (2003) E, L

88. Johansson, S., Litzén, U., Lundberg, H., Zhang, Z., ApJ 584, p. L107-L110 (2003) E, L

89. Johansson, S., Derkatch, A., Donnelly, M. P., Hartman, H., Hibbert, A., Karlsson, H., Kock, M., Li, Z. S., Leckrone, D. S., Litzén, U., Lundberg, H., Mannervik, S., Norlin, L.-O., Nilsson, H., Pickering, J., Raassen, T., Rostohar, D., Royen, P., Schmitt, A., Johanning, M., Sikström, C. M., Smith, P. L., Svanberg, S., Wahlgren, G. M., Phys. Scr. T100, 71 (2002) CM

90. Jonauskas, V., Bogdanovich, P., Keenan, F. P., Foord, M. E., Heeter, R. F., Rose, S. J., Ferland, G. J., Kisielius, R., van Hoof, P. A. M., Norrington, P. H., A\&A 433, 745-750 (2005) Q, QF

91. Jonauskas, V., Keenan, F. P., Foord, M. E., Heeter, R. F., Rose, S. J., Ferland, G. J., Kisielius, R., van Hoof, P. A. M., Norrington, P. H., A\&A 424, 363-369 (2004) Q, QF 92. Jonauskas, V., Keenan, F. P., Foord, M. E., Heeter, R. F., Rose, S. J., van Hoof, P. A. M., Ferland, G. J., Aggarwal, K. M., Kisielius, R., Norrington, P. H., A\&A 416, 383-389 (2004) Q, QF

93. Karpuškienè, R., Bogdanovich, P., Udris, A., At. Data Nucl. Data Tables 89, 45-76 (2005) Q

94. Karpuškiené, R., Bogdanovich, P., Udris, A., J. Phys. B 37, 2067-2086 (2004) Q

95. Kingston, A. E., Norrington, P. H., Boone, A. W., J. Phys. B 35, 4077-4100 (2002) $\mathrm{Q}, \mathrm{QF}$

96. Kisielius, R., Hibbert, A., Ferland, G. J., Foord, M. E., Rose, S. J., van Hoof, P. A. M., Keenan, F. P., MNRAS 344, 696-706 (2003) Q

97. Koc, K., J. Phys. B 37, 3821-3835 (2004) Q

98. Kubkowska, M. K., Szcześniak, T. M., Grycuk, T., Acta Phys. Pol. A 105, 317-328 (2004) A

99. Landi, E., A\&A 434, 365-376 (2005) Q, QF

100. Landi, E., Bhatia, A. K., At. Data Nucl. Data Tables 89, 195-265 (2005) Q, QF

101. Landi, E., Storey, P. J., Zeippen, C. J., ApJ 607, 640-652 (2004) Q, QF

102. Landi, E., Bhatia, A. K., ApJ 589, 1075-1084 (2003) Q, QF

103. Landi, E., Bhatia, A. K., ApJS 149, 251-263 (2003) Q, QF

104. Lawler, J. E., Sneden, C., Cowan, J. J., ApJ 604, 850-860 (2004) E

105. Lawler, J. E., Wahlgren, G. M., Wiese, W. L.: editors, Phys. Scr. T119, 82 pp. (2005) 106. Liang, G.-Y., Dong, G.-X., Zeng, J.-L., At. Data Nucl. Data Tables 88, 83161 (2004) Q, QF

107. Liu, X.-W., Wang, Z.-W., Phys. Rev. A 40, 1838-1842 (1989) Q 
108. Majumder, S., Gopakumar, G., Chaudhuri, R. K., Das, B. P., Merlitz, H., Mahapatra, U. S., Mukherjee, D., Eur. Phys. J. D 28, 3-9 (2004) QF

109. Majumder, S., Merlitz, H., Gopakumar, G., Das, B. P., Mahapatra, U. S., Mukherjee, D., ApJ 574, 513-517 (2002) Q

110. Mayo, R., Ortiz, M., Campos, J., JQSRT 94, 109-116 (2005) E

111. Mayo, R., Ortiz, M., Campos, J., Blagoev, K., Malcheva, G., Phys. Scr. 72, 142-147 (2005) E, Q

112. McKeown, K., Aggarwal, K. M., Keenan, F. P., Rose, S. J., Phys. Scr. 70, 295-303 (2004) Q, QF

113. Meléndez, M., Bautista, M. A., A\&A 436, 1123-1130 (2005) QF

114. Mohan, M., Singh, A. K., Jha, A. K. S., Singh, N., Pramana 65, 75-84 (2005) Q

115. Mulye, Y. G., Natarajan, L., Phys. Scr. 69, 24-29 (2004) Q

116. Murakami, I., Safronova, U. I., Vasilyev, A. A., Kato, T., At. Data Nucl. Data Tables 90, 1-74 (2005) Q

117. Murakami, I., Safronova, U. I., Kato, T., Can. J. Phys. 80, 1525-1542 (2002) Q

118. Nahar, S. N., Pradhan, A. K., A\&A 437, 345-354 (2005) Q

119. Nahar, S. N., A\&A 413, 779-787 (2004) Q, QF

120. Nahar, S. N., Eissner, W., Chen, G.-X., Pradhan, A. K., A\&A 408, 789-801 (2003) $\mathrm{Q}, \mathrm{QF}$

121. Natarajan, L., J. Phys. B 35, 3179-3190 (2002) Q

122. Nave, G., JOSA B 20, 2193-2202 (2003) E

123. Neerja, Gupta, G. P., Tripathi, A. N., Msezane, A. Z., At. Data Nucl. Data Tables 84, 85-97 (2003); Erratum: 85, 495 (2003) Q

124. Nilsson, H., Pickering, J. C., Phys. Scr. 67, 223-233 (2003) Q

125. Özdemir, L., Boya, G., JQSRT 91, 111-120 (2005) Q

126. Palmeri, P., Mendoza, C., Kallman, T. R., Bautista, M. A., Meléndez, M., A\&A 410, 359-364 (2003) Q

127. Palmeri, P., Mendoza, C., Kallman, T. R., Bautista, M. A., A\&A 403, 1175-1184 (2003) Q, QF

128. Pickering, J. C., Donnelly, M. P., Nilsson, H., Hibbert, A., Johansson, S., A\&A 396, 715-722 (2002) E, Q

129. Podobedova, L. I., Fuhr, J. R., Reader, J., Wiese, W. L., J. Phys. Chem. Ref. Data $33,525-540$ (2004) CP

130. Podobedova, L. I., Kelleher, D. E., Reader, J., Wiese, W. L., J. Phys. Chem. Ref. Data 33, 495-524 (2004) CP

131. Podobedova, L. I., Kelleher, D. E., Reader, J., Wiese, W. L., J. Phys. Chem. Ref. Data 33, 471-494 (2004) CP

132. Podobedova, L. I., Musgrove, A., Kelleher, D. E., Reader, J., Wiese, W. L., J. Phys. Chem. Ref. Data 32, 1367-1386 (2003) CP

133. Quinet, P., Biémont, E., MNRAS 340, 463-467 (2003) Q

134. Ray, H., AS\&S 283, 415-436 (2003) QF

135. Ray, H., ApJ 579, 914-919 (2002) QF

136. Ray, H., A\&A 391, 1173-1184 (2002) QF

137. Ray, H., J. Phys. B 35, p. L299-L307 (2002) QF

138. Rostohar, D., Derkatch, A., Hartman, H., Norlin, L.-O., Royen, P., Schef, P.,

Mannervik, S., Hyperfine Interact. 146-147, 151-159 (2003) LF

139. Safronova, U. I., Sataka, M., Albritton, J. R., Johnson, W. R., Safronova, M. S., At. Data Nucl. Data Tables 84, 1-83 (2003) Q

140. Savukov, I. M., Johnson, W. R., Safronova, U. I., At. Data Nucl. Data Tables 85, 83-167 (2003) Q, QF 
141. Schectman, R. M., Federman, S. R., Brown, M., Cheng, S., Fritts, M. C., Irving, R. E., Gibson, N. D., ApJ 621, 1159-1162 (2005) E, L

142. Schnabel, R., Schultz-Johanning, M., Kock, M., A\&A 414, 1169-1176 (2004) E, L

143. Scholl, T. J., Holt, R. A., Masterman, D., Rivest, R. C., Rosner, S. D., Sharikova, A., Can. J. Phys. 80, 1621-1629 (2002) L

144. Scholl, T. J., Holt, R. A., Masterman, D., Rivest, R. C., Rosner, S. D., Sharikova, A., Can. J. Phys. 80, 713-722 (2002) L

145. Singh, N., Singh, A. K., Mohan, M., Can. J. Phys. 81, 861-867 (2003) Q

146. Stone, P. M., Kim, Y.-K., J. Res. NIST 109, 505-515 (2004) Q

147. Tayal, S. S., A\&A 426, 717-720 (2004) Q

148. Tayal, S. S., ApJS 146, 459-465 (2003) Q

149. Tayal, S. S., J. Phys. B 36, 3239-3249 (2003) Q

150. Tayal, V., Gupta, G. P., Msezane, A. Z., Phys. Scr. 71, 627-637 (2005) Q

151. Toner, A., Hibbert, A., MNRAS 361, 673-678 (2005) Q

152. Träbert, E., Saathoff, G., Wolf, A., Eur. Phys. J. D 30, 297-302 (2004) LF

153. Träbert, E., Hyperfine Interact. 146-147, 269-273 (2003) LF

154. Tully, J. A., Chidichimo, M. C., J. Phys. B 37, 689-701 (2004) Q

155. Vilkas, M. J., Ishikawa, Y., Träbert, E., Phys. Scr. 72, 181-199 (2005) Q

156. Vilkas, M. J., Ishikawa, Y., Phys. Rev. A 69, 062503 (2004) Q, QF

157. Vilkas, M. J., Ishikawa, Y., J. Phys. B 36, 4641-4650 (2003) QF

158. Xu, H., Jiang, Z., Zhang, Z., Dai, Z., Svanberg, S., Quinet, P., Biémont, E., J. Phys. B 36, 1771-1781 (2003) L, Q

159. Xu, H., Jiang, Z., Svanberg, S., J. Phys. B 36, 411-417 (2003) L

160. Xu, H. L., Persson, A., Svanberg, S., Blagoev, K., Malcheva, G., Pentchev, V., Biémont, E., Campos, J., Ortiz, M., Mayo, R., Phys. Rev. A 70, 042508 (2004) E, L, Q 161. Xu, H. L., Svanberg, S., Cowan, R. D., Lefèbvre, P.-H., Quinet, P., Biémont, E., MNRAS 346, 433-440 (2003) L, Q

162. Xu, H. L., Svanberg, S., Quinet, P., Garnir, H. P., Biémont, E., J. Phys. B 36, 4773-4787 (2003) L, Q

163. Xu, H. L., Persson, A., Svanberg, S., Eur. Phys. J. D 23, 233-236 (2003) L

164. Xu, H.-L., Jiang, Z.-K., Svanberg, S., Phys. Scr. 67, 64-67 (2003) L

165. Yamamoto, N., Kato, T., Rosmej, F. B., JQSRT 96, 343-361 (2005) Q

166. Young, P. R., A\&A 417, 785-792 (2004) Q, QF

167. Zatsarinny, O., Bartschat, K., Bandurina, L., Gedeon, V., Phys. Rev. A 71, 042702 (2005) Q

168. Zatsarinny, O., Froese Fischer, C., J. Phys. B 35, 4669-4683 (2002) Q

169. Zeng, J., Yuan, J., J. Phys. B 35, 3041-3054 (2002) Q

170. Zeng, J.-L., Liang, G. Y., Zhao, G., Shi, J. R., MNRAS 357, 440-448 (2005) Q

171. Zeng, J.-L., Dong, G.-X., Zhao, G., Yuan, J.-M., J. Phys. B 37, 2529-2542 (2004) Q

172. Zeng, J.-L., Jin, F.-T., Zhao, G., Yuan, J.-M., J. Phys. B 36, 3457-3465 (2003) Q

173. Jiaolong Zeng, Jianmin Yuan, Phys. Rev. A 66, 022715 (2002) Q

174. Zhang, H. L., Sampson, D. H., At. Data Nucl. Data Tables 82, 357-389 (2002) Q

175. Zhang, Z. G., Svanberg, S., Palmeri, P., Quinet, P., Biémont, E., MNRAS 334, 1 (2002) L, Q

176. Zheng, N., Wang, T., ApJS 143, 231-240 (2002) Q

177. Zheng, N. W., Fan, J., Ma, D. X., Wang, T., J. Phys. Soc. Jpn. 72, 3091-3096 (2003) Q

178. Zheng, N. W., Wang, T., Spectrochim. Acta, Part B 58, 1319-1324 (2003) Q

179. Zheng, N. W., Wang, T., Chem. Phys. 282, 31-36 (2002) Q

180. Zheng, N. W., Wang, T., Zhou, T., Ma, D. X., J. Phys. Soc. Jpn. 71, 1672 (2002) Q 
181. Zhong, J.-Y., Zhang, J., Zhao, G., Lu, X., Chin. Astron. Astrophys. 28, 264-272 (2004) Q

182. Zielińska, S., Bratasz, L., Dzierżega, K., Phys. Scr. 66, 454-457 (2002) E

\section{WORKING GROUP ON Collision Processes PRESIDENT: D.R. Schultz and P.C. Stancil}

Since the last working group report in 2002, very active research continues that spans the vast range of atomic and molecular collision processes of interest in astrophysics. Given the large volume and scope of the pertinent published literature and the limited space available here, rather than seeking to comprehensively review this progress, we have attempted to identify works and some compilations of particular importance. In addition, access to an on-line bibliographic database that can be searched by collision reaction category, reactants, authors, and dates is made available by the Oak Ridge National Laboratory Controlled Fusion Atomic Data Center (CFADC 2005). Since a very high level of overlap exists between the atomic and molecular data needs of plasma science relevant to fusion energy and to astrophysics, this database, containing entries from 1978 to present, should be quite useful for those seeking such information. In addition, categorized bibliographies based largely on the bibliographic data compilation of the ORNL CFADC have been published in hardcopy by the International Atomic Energy Agency (CIAMDA 1998).

Here we organize our report summarizing new work on relevant collision processes including electron impact on atoms, ions, molecules, and molecular ions; ion-atom and atom-atom collisions; ion-, atom-, and molecule-molecule collisions; and reactive scattering and chemistry.

\subsection{Electron Collisions with Atoms, Ions, Molecules, and Molecular Ions}

Collisions of electrons with atoms, ions, molecules, and molecular ions are the major excitation mechanism in a wide range of astrophysical environments. In addition, they largely drive ionization and recombination, contribute to molecular fragmentation and formation, and influence transport. In the following sections we summarize recent work of interest for electron collisions with astrophysically relevant species regarding elastic scattering, excitation, dissociation, ionization, recombination, and electron detachment from negative ion since the time of the last Commission report (Schultz \& Stancil 2002).

\subsection{Electron-Atom Scattering}

New references for elastic scattering from atoms are as follows: H (Papp \& Hu 2002, James, Childers \& Khakoo 2004, Witthoeft, Loch \& Pindzola 2004, Jablonski, Salvat \& Powell 2004), noble gases (Garcia et al. 2002-Ne, Ar, Kr, Xe; Baek \& Grosswendt 2003 - He, Ne, Ar; Demesie et al. 2003-Xe; Blanco \& Garcia 2003-He, Ne, Ar, Kr, Xe, Date, Ishimaru \& Shimozuma 2003-Xe; Salvat 2003-He, Ne, Ar, Kr, Xe; McEachran \& Stauffer 2003-Kr; Ariyasinghe \& Goains 2004-Kr, Xe; Cho et al. 2004b-Kr; Milosavljevic, Sevic \& Marinkovic 2004-Kr), O (Plummer, Noble \& Le Dourneuf 2004), Mg (Al-Mulla \& Joensson 2002, Bartschat \& Sadeghpour 2003, Bartschat et al. 2004), Al (Jablonski, Salvat \& Powell 2004), Ca (Bartschat \& Sadeghpour 2003), Ni (Jablonski, Salvat \& Powell 2004), and Zn (Sienkiewicz et al. 2002). 
Excitation of atoms are covered in new references: H (Sweeney et al. 2001, Kim 2001, James et al. 2002, Sweeney, Grafe \& Shyn 2004, James, Childers. \& Khakoo 2004, Witthoeft, Loch. \& Pindzola 2004), He (Kim 2001, Dogan \& Crowe 2002, Mityureva, Smirnov \& Ponomarenko 2002, Bartschat \& Andersen 2003, Baek \& Grosswendt 2003, Harries, Hammond \& Murray 2003, Merabet et al. 2003b, Kheifets 2004), Li (Kim 2001, Griffin et al. 2001, Witthoeft et al. 2003), Be (Kim 2001, Ballance et al. 2003b), C (Srivastava, McEachran \& Stauffer 2002, Kim \& Desclaux 2002, Zatsarinny \& Fischer 2002), N (Kim \& Desclaux 2002), O (Zatsarinny \& Tayal 2002a, Kim \& Desclaux 2002, Tayal 2002, Johnson et al. 2003a, Johnson et al. 2003b, Plummer, Noble \& Le Dourneuf 2004), Ne (Boffard et al. 2001, Khakoo et al. 2002, Stewart et al. 2002, Meneses et al. 2002, Baek \& Grosswendt 2003, Zatsarinny \& Bartschat 2004a, Ballance \& Griffin 2004), Mg (Fursa \& Bray 2001, Kim 2001, Kai et al. 2004), Si (Srivastava, McEachran. \& Stauffer 2002), S (Zatsarinny \& Tayal 2002b), Ar (Stewart et al. 2002, Baek \& Grosswendt 2003, Weber, Boffard \& Lin 2003, Madison et al. 2004, Zatsarinny \& Bartschat 2004b), Ca (Kim 2001, Muktavat, Srivastava \& Stauffer 2002), Kr (Stewart et al. 2002, Yuan et al. 2002, Tsurubuchi Kobayashi \& Hyodo 2003, Li et al. 2003, Yu et al. 2003, Cho et al. 2004b), and Xe (Kanik, Johnson \& James 2001, Boechat-Roberty et al. 2002, Yu et al. 2003, Date, Ishimaru \& Shimozuma 2003, Bartschat, Dasgupta \& Madison 2004).

New publications on ionization include: H (Deb \& Crothers 2002, Lepp, Stancil \& Dalgarno 2002, Isaacs et al. 2001, Szluniska, Van Reeth \& Laricchia 2002, Bartlett, Stelbovics \& Bray 2003, Childers et al. 2003, Bartlett \& Stelbovics 2004, Chen et al. 2004, Childers et al. 2004), He (Lepp, Stancil \& Dalgarno 2002, Dogan \& Crowe 2002, Denifl et al. 2002, Keller 2003, Bray, Fursa \& Stelbovics 2003, Fursa \& Bray 2003, Merabet et al. 2003a, Defrance, Kereselidze \& Machavariani 2003, Merabet et al. 2003b, Kheifets \& Bray 2004, Sorokin et al. 2004, Kheifets 2004, Pindzola et al. 2004, Uddin et al. 2004b), noble gases (Rejoub, Lindsay \& Stebbings 2002 - He, Ne, Ar, Kr, Xe; Kobayashi et al. 2002 - Ne, Ar, Kr, Xe; Bartlett \& Stelbovics 2002 - Ne, Ar, Kr, Xe; Gstir et al. 2002 - Ne, Ar, Xe; Szluniska, Van Reeth \& Laricchia 2002 - He, Ne, Ar, Kr, Xe; Gstir et al. 2003 - He, Ne, Ar, Kr, Xe; Erwin \& Kunc 2003 - Ar, Kr, Xe, Rn), Li (Huang et al. 2002, Ghosh \& Sinha 2003), C (Kim \& Desclaux 2002, Szluniska, Van Reeth \& Laricchia 2002, Alfaz Uddin \& Basak 2003a), N (Kim \& Desclaux 2002, Szluniska, Van Reeth \& Laricchia 2002, Alfaz Uddin \& Basak 2003a), O (Kim \& Desclaux 2002, Szluniska, Van Reeth \& Laricchia 2002, Joshipura, Antony \& Vinodkumar 2002, Alfaz Uddin \& Basak 2003a), Mg (Kampp et al. 2002, Jha \& Roy 2002), Si (Bartlett \& Stelbovics 2002), P (Bartlett \& Stelbovics 2002), S (Bartlett \& Stelbovics 2002), Ar (Biava et al. 2002, Stano et al. 2003, Cooper \& van Boeyen 2004), Ca (Alfaz Uddin \& Basak 2003b), Ti (Alfaz Uddin \& Basak 2003b), Cr (Alfaz Uddin \& Basak 2003a), Mn (Llovet, Merlet \& Salvat 2002, Alfaz Uddin \& Basak 2003a), Fe (Llovet, Merlet \& Salvat 2002, Alfaz Uddin \& Basak 2003a), Kr (Denifl et al. 2002, Loch et al. 2002), Xe (Date, Ishimaru \& Shimozuma 2003), and series of atoms (Santos, Parente \& Kim 2003 - C, N, O, Ne, Mg, Al, Si, P, S, Ar, Ti, Cr, Fe, Co, Ni, Kr).

Negative ion formation (i.e. e $+\mathrm{A} \rightarrow \mathrm{A}^{-}$) has been studied for: $\mathrm{H}$ (Ghosh, Nath \& Sinha 2003, Grujic 2003, Fritioff et al. 2004), Li (Le Padellec et al. 2002), and S (Fritioff et al. 2003).

\subsection{Electron-Ion Scattering}

New elastic data exists for $\mathrm{He}^{+}$(Bhatia 2002).

New work for excitation of atomic ions include: $\mathrm{He}^{+}$(Kim 2002, Witthoeft, Pindzola \& Colgan 2003), He-like (Whiteford et al. 2001 - Ar, Fe; Zhang \& Sampson 2002 - O, 
Fe), $\mathrm{Li}^{+}$(Ballance et al. 2003a), Li-like (Whiteford et al. 2002 - Ar, Fe; Starobinets et al. 2003 - Be, B), Be-isonuclear (Ballance et al. 2003b-Be ${ }^{+-3+}$ ), $\mathrm{C}^{2+}$ (Mitnik et al. 2003), $\mathrm{C}^{3+}$ (Aggarwal \& Keenan 2004a), O ${ }^{2+}$ (Niimura, Smith \& Chutjian 2002), O ${ }^{3+}$ (Smith et al. 2003), $\mathrm{O}^{6+}$ (Delahaye \& Pradhan 2002), $\mathrm{O}^{7+}$ (Berrington \& Ballance 2002), $\mathrm{Ne}^{2+}$ (McLaughlin, Daw \& Bell 2002), $\mathrm{Ne}^{8+}$ (Bautista 2003), Ne-like (Brown, Beiersdorfer \& Widmann 2001 - Fe, Co, Ni, Kr), $\mathrm{Mg}^{+}$(Kim 2002), Mg-like (Kai, Srivastava \& Nakazaki 2004- S, Ar, Ca), Al ${ }^{2+}$ (Dunn et al. 2002, Bannister et al. 2002, Smith et al. 2003), $\mathrm{Si}^{6+}$ (Bhatia \& Landi 2003), $\mathrm{Si}^{2+}$ (Janzen et al. 2003, Smith et al. 2003), $\mathrm{P}^{2+}$ (Gupta \& Msezane 2002), $\mathrm{Ar}^{6+}$ (Smith et al. 2003), $\mathrm{Ar}^{7+}$ (Smith et al. 2003), $\mathrm{Ca}^{6+}$ (Landi \& Bhatia 2003), $\mathrm{Ca}^{7+}$ (Landi, Storey \& Zeippen 2004), $\mathrm{Ca}^{14+}$ (Aggarwal \& Keenan 2002, Aggarwal \& Keenan 2003b), $\mathrm{Ti}^{2+}$ (Popovic et al. 2002), $\mathrm{Fe}^{+}$(Burke et al. 2002, Ramsbottom et al. 2002, Ramsbottom et al. 2004), $\mathrm{Fe}^{2+}$ (McLaughlin et al. 2002), $\mathrm{Fe}^{4+}$ (Woeste et al. 2002), $\mathrm{Fe}^{8+}$ (Storey, Zeippen \& Le Dourneuf 2002), $\mathrm{Fe}^{10+}$ (Aggarwal \& Keenan 2003a), Fe ${ }^{12+}$ (Aggarwal \& Keenan 2004b, Aggarwal \& Keenan 2005), Fe ${ }^{14+}$ (Aggarwal, Keenan \& Msezane 2003), $\mathrm{Fe}^{16+}$ (Chen, Pradhan \& Eissner 2003), $\mathrm{Fe}^{21+}(\mathrm{Gu}$ 2004), $\mathrm{Fe}^{23+}$ (Pindzola 2002, Bautista et al. 2003), $\mathrm{Fe}^{25+}$ (Ballance, Badnell \& Berrington 2002), Fe-isonuclear (Bautista et al. $2004-\mathrm{Fe}^{16-22+}$ ), $\mathrm{Ni}^{+}$(Bautista 2004), $\mathrm{Ni}^{4+}$ (Burke et al. 2002), $\mathrm{Ni}^{24+}$ (Chidichimo, Badnell \& Tully 2003), $\mathrm{Zn}^{+}$(Kim 2002), and $\mathrm{Xe}^{26+}$ (Badnell et al. 2004).

Ionization and detachment have been studied for: $\mathrm{H}^{-}$(Ghosh, Nath \& Sinha 2003), H-like ions (Watanabe et al. 2003 - Fe, Mo; Alfaz Uddin et al. 2003 - He, Li, B, C, N, O, Ne, Fe; Bartlett \& Stelbovics $2004-\mathrm{Li}, \mathrm{Be}), \mathrm{He}^{+}$(Lepp, Stancil \& Dalgarno 2002), He-like ions (Kuo \& Huang 2001 - Li, B, C, N, Fe; Uddin et al. 2004b - Li, B, C, N, O, Ne, $\mathrm{Fe}$ ), $\mathrm{Li}^{+}$(Lepp, Stancil \& Dalgarno 2002), $\mathrm{Li}^{2+}$ (Lepp, Stancil \& Dalgarno 2002, Colgan, Pindzola \& Robicheaux 2002), Li-like ions (Uddin et al. 2004a - C, N, O, Ne), Be ions (Colgan et al. 2003b - Be ${ }^{+-3+}$ ), Be-like ions (Chang et al. $2004-\mathrm{B}, \mathrm{C}, \mathrm{N}, \mathrm{O}, \mathrm{Ne}, \mathrm{Al}, \mathrm{Ar}$, $\mathrm{Fe}$ ), $\mathrm{C}^{+}$(Jha 2002), $\mathrm{N}^{+}$(Jha 2002), $\mathrm{O}^{+}$(Jha 2002), $\mathrm{O}^{4+}$ (Uddin et al. 2004a), O ions (Loch et al. $2003-\mathrm{O}^{+-4+}$ ), $\mathrm{Ne}^{+}$(Jha 2002), $\mathrm{Ne}^{6+}$ (Uddin et al. 2004a), $\mathrm{Mg}^{+}$(Becker et al. 2004), Al ions (Aichele et al. $2001-\mathrm{Al}^{3-7+}$ ), Ar ions (Zhang et al. $2002-\mathrm{Ar}^{4-11+}$ ), $\mathrm{Ti}^{3+}$ (van Zoest et al. 2004), $\mathrm{Ti}^{11+}$ (Qi et al. 2002a), $\mathrm{Cr}^{13+}$ (Qi et al. 2002b), and $\mathrm{Kr}$ ions (Khouilid et al. $2001-\mathrm{Kr}^{12-18+}$; Loch et al. $2002-\mathrm{Kr}^{+-35+}$; Beigman, Defrance \& Vainshtein 2003a - Kr ${ }^{10-18+}$; Beigman, Defrance \& Vainshtein 2003b - Kr ${ }^{10-12,17,18+}$ ).

Another important process is recombination for which a number of new works have appeared: He ions (Lepp, Stancil \& Dalgarno 2002 - $\mathrm{He}^{+-2+}$ ), He-like ions (Zhang \& Sampson 2002 - O, Fe; O'Rourke et al. 2003 - Ti, Fe; Behar et al. 2004 - Ar, Fe), Li ions (Lepp, Stancil \& Dalgarno $2002-\mathrm{Li}^{+-3+}$; Colgan, Pindzola \& Badnell $2004-\mathrm{Be}, \mathrm{B}$, C, N, O, Ne, Mg, Al, Si, P, S, Ar, Ti, Cr, Fe, Ni, Kr, Xe and others), Be ${ }^{+}$(Mohamed et al. 2002, Schuch 2003), Be-like ions (Colgan et al. 2003a - B, C, O, N, Ne, Mg, Al, Si, P, S, Ar, Ca, Ti, Cr, Fe, Ni, Kr, Xe and others; Schnell et al. 2003 - F, Fe), B-like ions (Altun et al. 2004 - C, N, O, Ne, Mg, Al, Si, P, S, Ar, Ca, Ti, Cr, Fe, Ni, Kr, Xe and others), $\mathrm{C}^{3+}$ (Bureyeva et al. 2002b), C-like (Zatsarinny et al. 2004a - N, O, Ne, Mg, Al, Si, P, S, Ar, Ca, Ti, Cr, Fe, Co, Ni, Kr, Xe and others), N ${ }^{2+}$ (Kisielius \& Storey 2002), N-like ions (Mitnik \& Badnell 2004 - O, Ne, Mg, Al, Si, P, S, Ar, Ca, Ti, Cr, Fe, $\mathrm{Ni}, \mathrm{Kr}, \mathrm{Xe}$ and others), $\mathrm{O}^{5+}$ (Boehm et al. 2003, Murakami, Safronova \& Kato 2002), O ions (Badnell et al. $2003-\mathrm{O}^{4-5+}$ ), O-like ions (Chen $2002-\mathrm{Mg}$, S, Si, Fe; Zatsarinny et al. 2003 - Ne, Mg, Al, Si, P, S, Ar, Ca, Ti, Cr, Fe, Co, Ni, Kr, Xe and others), Ne-like ions (Zatsarinny et al. 2004b - Mg, Al, Si, P, S, Ar, Ca, Ti, Cr, Fe, Co, Ni, Kr, Xe and others), $\mathrm{Na}^{8+}$ (Nikolic et al. 2004), $\mathrm{Mg}^{+}$(Bureyeva et al. 2002b), $\mathrm{Mg}^{8+}$ (Schippers et al . 2004), Mg ions ( $\mathrm{Gu} 2003 \mathrm{a}-\mathrm{Mg}^{4-12+}$; Gu 2003b- $\mathrm{Mg}^{2-11+}$ ), $\mathrm{Si}^{14+}$ (Heerlein, Zwicknagel 
\& Toepffer 2002), Si ions ( Gu 2003a- $\left.\mathrm{Si}^{6-14+} ; \mathrm{Gu} 2003 \mathrm{~b}-\mathrm{Si}^{4-13+}\right)$, S ions (Gu 2003a - $\left.\mathrm{S}^{8-16+} ; \mathrm{Gu} 2003 \mathrm{~b}-\mathrm{S}^{6-15+}\right)$, Ar ions (Gu 2003a - Ar10-18+; Gu 2003b - Ar $\left.{ }^{8-17+}\right)$, Ca ions $\left(\mathrm{Gu} 2003 \mathrm{a}-\mathrm{Ca}^{12-20+} ; \mathrm{Gu} 2003 \mathrm{~b}-\mathrm{Ca}^{10-19+}\right), \mathrm{Ti}^{20+}$ (O'Rourke et al. 2004), $\mathrm{Fe}^{17+}$ (Gorczyca, Badnell \& Savin 2002, Zhang, Nahar \& Pradhan 2001), Fe ${ }^{18+}$ (Savin et al. 2002), $\mathrm{Fe}^{23+}$ (Pindzola 2002), Fe ions (Gu 2003a - Fe ${ }^{18-26+} ; \mathrm{Gu} 2003 \mathrm{~b}-\mathrm{Fe}^{16-25+}$; $\mathrm{Gu} 2003 \mathrm{c}-\mathrm{Fe}^{16-23+}$; Dasgupta \& Whitney $2004-\mathrm{Fe}^{24-25+}$ ), $\mathrm{Ni}^{17+}$ (Fogle et al. 2003a, Fogle et al. 2003b), Ni ions ( $\mathrm{Gu} 2003 \mathrm{a}-\mathrm{Ni}^{20-28+}$; Gu 2003b - Ni ${ }^{18-27+}$; Dasgupta \& Whitney $2004-\mathrm{Ni}^{26-27+}$ ), $\mathrm{Zn}^{27+}$ (Bureyeva et al. 2002a, Bureyeva et al. 2002b), Kr ${ }^{34+}$ (Madzunkov et al. 2002), and $\mathrm{Xe}^{27+}$ (Yan, Li \& Yao 2003).

\subsection{Electron-Molecule Scattering}

For molecules, new elastic scattering references have appeared as follows: $\mathrm{H}_{2}$ (Lee \& Mazon 2002), $\mathrm{H}_{2} \mathrm{O}$ (Cho et al. 2004a, Faure, Gorfinkiel \& Tennyson 2004), LiH (Antony et al. 2004), CH (Baluja \& Msezane 2001), $\mathrm{CH}_{4}$ (Ariyasinghe \& Powers 2002, Ariyasinghe, Wijeratne \& Palihawadana 2004), $\mathrm{C}_{2} \mathrm{H}_{2}$ (Ariyasinghe \& Powers 2002), $\mathrm{C}_{2} \mathrm{H}_{4}$ (Ariyasinghe \& Powers 2002, Panajotovic et al. 2003, Szmytkowski, Kwitnewski \& Ptasinska-Denga 2003, Brescansin et al. 2004, Trevisan, Orel \& Rescigno 2003), $\mathrm{C}_{2} \mathrm{H}_{6}$ (Ariyasinghe \& Powers 2002, Maia \& Bettega 2003), $\mathrm{C}_{3} \mathrm{H}_{4}$ (Nakano et al. 2002, Lopes \& Bettega 2003), $\mathrm{C}_{3} \mathrm{H}_{6}$ (Curik \& Gianturco 2002), $\mathrm{C}_{4} \mathrm{H}_{6}$ (Szmytkowski \& Kwitnewski 2003, Lopes et al. 2004), $\mathrm{C}_{6} \mathrm{H}_{6}$ (Makochekanwa, Sueoka \& Kimura 2003, Kimura, Makochekanwa \& Sueoka 2004, Makochekanwa, Sueoka \& Kimura 2004), CO (Blanco \& Garcia 2003), $\mathrm{CO}_{2}$ (Gianturco \& Stoecklin 2001, Allan, M. 2002, Blanco \& Garcia 2003), CS 2 (Allan, M. 2003), NO (Zhang et al. 2004), $\mathrm{N}_{2}$ (Blanco \& Garcia 2003), $\mathrm{N}_{2} \mathrm{O}$ (Lee et al. 2002a, Akther et al. 2002, Allan \& Skalicky 2003), O 2 (Garcia, Blanco \& Williart 2001, Linert, Zubek \& Zubek 2004), $\mathrm{O}_{3}$ (de Pablos et al. 2002), OH (Sobrinho, Lozano \& Lee 2004), OCS (Bettega, Lima \& Ferreira 2004), SiH (Lee et al. 2002b), $\mathrm{SiH}_{4}$ (Ariyasinghe, Wijerathna \& Powers 2003), $\mathrm{Si}_{2} \mathrm{H}_{6}$ (Maia \& Bettega 2003), $\mathrm{PH}_{2}$ (Bettega \& Lima 2004), $\mathrm{PH}_{3}$ (Ariyasinghe, Wijerathna \& Powers 2003, Szmytkowski et al. 2004), and SH (Baluja \& Msezane 2002).

Regarding excitation, new references include: $\mathrm{H}_{2}$ (Lee \& Mazon 2002, Pigarov 2002, Celiberto, Capitelli \& Laricchiuta 2002, Machado et al. 2001, Trevisan \& Tennyson 2001, Wrkich et al. 2002, Curik \& Carsky 2003, Harries, Hammond \& Murray 2003, Liu et al. 2003, Horacek et al. 2004, da Costa, da Paixao \& Lima 2004, Laricchiuta, Celiberto \& Janev 2004), $\mathrm{H}_{2} \mathrm{O}$ (Allan \& Moreira 2002, Curik \& Carsky 2003, Itikawa 2004, Faure, Gorfinkiel \& Tennyson 2004), LiH (Antony et al. 2004), CH (Baluja \& Msezane 2001), $\mathrm{CH}_{4}$ (Nishimura \& Gianturco 2002, Itikawa 2004, Joshipura et al. 2004), $\mathrm{C}_{2} \mathrm{H}_{4}$ (Panajotovic et al. 2003, Szmytkowski, Kwitnewski \& Ptasinska-Denga 2003), $\mathrm{C}_{2} \mathrm{H}_{6}$ (Merz \& Linder 2003a, Itikawa 2004), $\mathrm{C}_{3} \mathrm{H}_{4}$ (Nakano et al. 2002), $\mathrm{C}_{4} \mathrm{H}_{6}$ (Szmytkowski \& Kwitnewski 2003), $\mathrm{C}_{6} \mathrm{H}_{6}$ (Makochekanwa, Sueoka \& Kimura 2003), $\mathrm{CH}_{3} \mathrm{CH}_{2} \mathrm{CH}_{3}(\mathrm{Merz}$ \& Linder 2003b), hydrocarbons (Janev \& Reiter $2003-\mathrm{CH}, \mathrm{CH}_{2}, \mathrm{CH}_{3}, \mathrm{CH}_{4}, \mathrm{C}_{2} \mathrm{H}, \mathrm{C}_{2} \mathrm{H}_{2}$, $\left.\mathrm{C}_{2} \mathrm{H}_{3}, \mathrm{C}_{2} \mathrm{H}_{4}, \mathrm{C}_{2} \mathrm{H}_{5}, \mathrm{C}_{2} \mathrm{H}_{6}, \mathrm{C}_{3} \mathrm{H}, \mathrm{C}_{3} \mathrm{H}_{2}, \mathrm{C}_{3} \mathrm{H}_{3}, \mathrm{C}_{3} \mathrm{H}_{4}, \mathrm{C}_{3} \mathrm{H}_{5}, \mathrm{C}_{3} \mathrm{H}_{6}, \mathrm{C}_{3} \mathrm{H}_{7}, \mathrm{C}_{3} \mathrm{H}_{8}\right), \mathrm{CS}_{2}$ (Allan 2003, Michelin et al. 2003, Kroin et al. 2003), CO (Poparic, Vicic \& Belic 2001, Kroin, Michelin \& Lee 2001, Michelin et al. 2003), $\mathrm{CO}_{2}$ (Rescigno et al. 2002, Green et al. 2002a, Rocha \& Bielschowsky 2002, Allan 2002, McCurdy et al. 2003, Michelin et al. 2003, Kroin et al. 2003, Itikawa 2004, Vanroose et al. 2004), $\mathrm{N}_{2}$ (Poparic, Vicic \& Belic 2002, Feng, Sun \& Morrison 2003), NO (Schappe, Edgell \& Urban 2002), $\mathrm{N}_{2} \mathrm{O}$ (Akther et al. 2002, Allan \& Skalicky 2003), O (Johnson \& Kanik 2001, Green et al. 2002b, Terrell, Hansen \& Ajello 2004), $\mathrm{O}_{3}$ (de Pablos et al. 2002), OCS (Michelin et al. 2003, Kroin et al. 2003), $\mathrm{SiH}_{4}$ (Ariyasinghe, Wijerathna \& Powers 2003, Joshipura et al. 2004), $\mathrm{PH}_{3}$ (Ariyasinghe, Wijerathna \& Powers 2003), and SH (Baluja \& Msezane 2002). 
New work for dissociative processes consist of the following: $\mathrm{H}_{2}$ (Harb, Kedzierski \& McConkey. 2000, Trevisan \& Tennyson 2001, Krasheninnikov 2002, Pigarov 2002, Celiberto, Capitelli \& Laricchiuta 2002, Fabrikant, Wadehra \& Xu 2002, Horacek et al. 2004, Laricchiuta, Celiberto \& Janev 2004), $\mathrm{H}_{2} \mathrm{O}$ (Haxton et al. 2004), $\mathrm{CH}_{4}$ (Luna et al. 2003), $\mathrm{C}_{2} \mathrm{H}_{4}$ (Szmytkowski, Kwitnewski \& Ptasinska-Denga 2003), $\mathrm{C}_{4} \mathrm{H}_{6}$ (Szmytkowski \& Kwitnewski 2003), $\mathrm{C}_{6} \mathrm{H}_{6}$ (Makochekanwa, Sueoka \& Kimura 2003), $\mathrm{CH}_{3} \mathrm{OH}$ (Skalicky \& Allan 2004), hydrocarbons (Janev \& Reiter $2003-\mathrm{CH}, \mathrm{CH}_{2}, \mathrm{CH}_{3}, \mathrm{CH}_{4}, \mathrm{C}_{2} \mathrm{H}, \mathrm{C}_{2} \mathrm{H}_{2}$, $\left.\mathrm{C}_{2} \mathrm{H}_{3}, \mathrm{C}_{2} \mathrm{H}_{4}, \mathrm{C}_{2} \mathrm{H}_{5}, \mathrm{C}_{2} \mathrm{H}_{6}, \mathrm{C}_{3} \mathrm{H}, \mathrm{C}_{3} \mathrm{H}_{2}, \mathrm{C}_{3} \mathrm{H}_{3}, \mathrm{C}_{3} \mathrm{H}_{4}, \mathrm{C}_{3} \mathrm{H}_{5}, \mathrm{C}_{3} \mathrm{H}_{6}, \mathrm{C}_{3} \mathrm{H}_{7}, \mathrm{C}_{3} \mathrm{H}_{8}\right), \mathrm{CS}_{2}$ (Kedzierski 2002), NO (Allan 2004), $\mathrm{N}_{2} \mathrm{O}$ (Allan \& Skalicky 2003), and $\mathrm{O}_{3}$ (de Pablos et al. 2002).

Research on molecular ionization have been performed on: $\mathrm{H}_{2}$ (Hanel et al. 2002, Pigarov 2002, Celiberto, Capitelli \& Laricchiuta 2002, Houamer et al. 2003, Mansouri et al. 2004, Liu \& Shemansky 2004), $\mathrm{H}_{2} \mathrm{O}$ (Champion, Hanssen \& Hervieux 2002a, Hanel et al. 2002, Champion, Hanssen \& Hervieux 2002b, Vinodkumar et al. 2003 - crystalline ice; Champion, Hanssen \& Hervieux 2004), $\mathrm{H}_{2} \mathrm{~S}$ (Lindsay, Rejoub \& Stebbings 2003), $\mathrm{B}_{2} \mathrm{H}_{6}$ (Basner, Schmidt \& Becker 2003), $\mathrm{CH}_{4}$ (Stano et al. 2003, Luna et al. 2003, Joshipura et al. 2004, Champion, Hanssen \& Hervieux 2004), $\mathrm{C}_{2} \mathrm{H}_{4}$ (Szmytkowski, Kwitnewski \& Ptasinska-Denga 2003), $\mathrm{C}_{6} \mathrm{H}_{6}$ (Hanel et al. 2002, Makochekanwa, Sueoka \& Kimura 2003), $\mathrm{CH}_{3} \mathrm{COCOCH}_{3}$ (Takahashi et al. 2003), hydrocarbons (Janev \& Reiter $2003-\mathrm{CH}, \mathrm{CH}_{2}, \mathrm{CH}_{3}, \mathrm{CH}_{4}, \mathrm{C}_{2} \mathrm{H}, \mathrm{C}_{2} \mathrm{H}_{2}, \mathrm{C}_{2} \mathrm{H}_{3}, \mathrm{C}_{2} \mathrm{H}_{4}, \mathrm{C}_{2} \mathrm{H}_{5}, \mathrm{C}_{2} \mathrm{H}_{6}, \mathrm{C}_{3} \mathrm{H}, \mathrm{C}_{3} \mathrm{H}_{2}$, $\mathrm{C}_{3} \mathrm{H}_{3}, \mathrm{C}_{3} \mathrm{H}_{4}, \mathrm{C}_{3} \mathrm{H}_{5}, \mathrm{C}_{3} \mathrm{H}_{6}, \mathrm{C}_{3} \mathrm{H}_{7}, \mathrm{C}_{3} \mathrm{H}_{8}$ ), $\mathrm{CO}$ (Hudson, Vallance \& Harland 2004), $\mathrm{CO}_{2}$ (Hudson, Vallance \& Harland 2004), $\mathrm{CS}_{2}$ (Lindsay, Rejoub \& Stebbings 2003, Hudson, Vallance \& Harland 2004), $\mathrm{NH}_{3}$ (Champion, Hanssen \& Hervieux 2004), $\mathrm{N}_{2} \mathrm{O}$ (Lindsay, Rejoub \& Stebbings 2003), $\mathrm{NO}_{2}$ (Jiao, DeJoseph \& Garscadden 2002), $\mathrm{O}_{2}$ (Johnson \& Kanik 2001, Joshipura, Antony \& Vinodkumar 2002), $\mathrm{O}_{3}$ (Joshipura, Antony \& Vinodkumar 2002), $\mathrm{O}_{4}$ (Joshipura, Antony \& Vinodkumar 2002), OCS (Hudson, Vallance \& Harland 2004), OHCCHO (Takahashi et al. 2003), $\mathrm{SiH}_{3}$ (Ariyasinghe, Wijerathna \& Powers 2003), $\mathrm{SiH}_{4}$ (Joshipura et al. 2004), and $\mathrm{PH}_{3}$ (Ariyasinghe, Wijerathna \& Powers 2003).

Andersen, Herber \& Zajfman (2004) have studied negative ion formation from $\mathrm{C}_{2}$.

\subsection{Electron-Molecular-Ion Scattering}

New elastic scattering results exist for $\mathrm{H}_{3}^{+}$and $\mathrm{H}_{3} \mathrm{O}^{+}$(Faure \& Tennyson 2002a).

For excitation, studies have been conducted for: $\mathrm{H}_{2}^{+}$(Pigarov 2002), $\mathrm{H}_{3}^{+}$(Faure \& Tennyson 2002a, Faure \& Tennyson 2002b, Gorfinkiel \& Tennyson 2004), $\mathrm{H}_{3} \mathrm{O}^{+}$(Faure \& Tennyson 2002a, Faure \& Tennyson 2002b), and hydrocarbon ions (Janev \& Reiter $2003-\mathrm{CH}^{+}, \mathrm{CH}_{2}^{+}, \mathrm{CH}_{3}^{+}, \mathrm{CH}_{4}^{+}, \mathrm{C}_{2} \mathrm{H}^{+}, \mathrm{C}_{2} \mathrm{H}_{2}^{+}, \mathrm{C}_{2} \mathrm{H}_{3}^{+}, \mathrm{C}_{2} \mathrm{H}_{4}^{+}, \mathrm{C}_{2} \mathrm{H}_{5}^{+}, \mathrm{C}_{2} \mathrm{H}_{6}^{+}, \mathrm{C}_{3} \mathrm{H}^{+}, \mathrm{C}_{3} \mathrm{H}_{2}^{+}$, $\left.\mathrm{C}_{3} \mathrm{H}_{3}^{+}, \mathrm{C}_{3} \mathrm{H}_{4}^{+}, \mathrm{C}_{3} \mathrm{H}_{5}^{+}, \mathrm{C}_{3} \mathrm{H}_{6}^{+}, \mathrm{C}_{3} \mathrm{H}_{7}^{+}, \mathrm{C}_{3} \mathrm{H}_{8}^{+}\right)$.

References on dissociative processes have appeared for: $\mathrm{H}_{2}^{+}$(Neau et al. 2002, Krasheninnikov 2002, Pigarov 2002, Takagi 2002, Strasser et al. 2002b, Florescu et al. 2003, AlKhalili et al. 2003, Abdellahi El Ghazaly et al. 2004), $\mathrm{H}_{3}^{+}$(Krasheninnikov 2002, Glosik et al. 2001, Strasser et al. 2002a, McCall et al. 2004, Royal, Larson \& Orel 2004, Gorfinkiel \& Tennyson 2004, Kalhori et al. 2004), $\mathrm{HeH}^{+}$(Royal, Larson \& Orel 2004, Takagi 2004), $\mathrm{H}_{2} \mathrm{O}^{+}$(Thomas et al. 2002), $\mathrm{CH}^{+}$(Bannister et al. 2003), $\mathrm{CH}_{4}^{+}$(Janev 2002), $\mathrm{C}_{2} \mathrm{H}_{2}^{+}$(Derkatch, Minaev \& Larsson 2003), $\mathrm{C}_{2} \mathrm{H}_{4}^{+}$(Janev 2002), ${ }_{2} \mathrm{H}_{6}^{+}$(Janev 2002), $\mathrm{C}_{3} \mathrm{H}_{8}^{+}$(Janev 2002), $\mathrm{CO}_{2}^{+}$(Bahati et al. 2001a, Seiersen et al. 2003), $\mathrm{N}_{2}^{+}$(Bahati, E. M. et al. 2001b), and $\mathrm{SO}_{2}^{+}$(Geppert et al. 2004).

New reports of results for ionization include: $\mathrm{H}_{2}^{+}$(Serov et al. 2002), $\mathrm{H}_{3}^{+}$(Gorfinkiel \& Tennyson 2004), hydrocarbon ions (Janev \& Reiter $2003-\mathrm{CH}^{+}, \mathrm{CH}_{2}^{+}, \mathrm{CH}_{3}^{+}, \mathrm{CH}_{4}^{+}$, $\mathrm{C}_{2} \mathrm{H}^{+}, \mathrm{C}_{2} \mathrm{H}_{2}^{+}, \mathrm{C}_{2} \mathrm{H}_{3}^{+}, \mathrm{C}_{2} \mathrm{H}_{4}^{+}, \mathrm{C}_{2} \mathrm{H}_{5}^{+}, \mathrm{C}_{2} \mathrm{H}_{6}^{+}, \mathrm{C}_{3} \mathrm{H}^{+}, \mathrm{C}_{3} \mathrm{H}_{2}^{+}, \mathrm{C}_{3} \mathrm{H}_{3}^{+}, \mathrm{C}_{3} \mathrm{H}_{4}^{+}, \mathrm{C}_{3} \mathrm{H}_{5}^{+}, \mathrm{C}_{3} \mathrm{H}_{6}^{+}$, 
$\mathrm{C}_{3} \mathrm{H}_{7}^{+}, \mathrm{C}_{3} \mathrm{H}_{8}^{+}$), $\mathrm{CO}_{2}^{+}$(Bahati et al. 2001a, Deutsch et al. 2002), and $\mathrm{N}_{2}^{+}$(Deutsch et al. 2002, Bahati et al. 2001b).

Finally, recombination of electrons with molecular ions have been studied for: $\mathrm{H}_{2}^{+}$ (Neau et al. 2002, Krasheninnikov 2002, Pigarov 2002, Takagi 2002, Florescu et al. 2003, Ngassam et al. 2003, Al-Khalili et al. 2003), $\mathrm{H}_{3}^{+}$(Glosik et al. 2001, Strasser et al. 2002a, Strasser et al. 2002b, Kokoouline \& Greene 2003a,2003b, McCall et al. 2004, Royal, Larson \& Orel 2004), $\mathrm{H}_{2} \mathrm{O}^{+}$(Thomas et al. 2002), $\mathrm{HeH}^{+}$(Royal, Larson \& Orel 2004, Takagi 2004), hydrocarbon molecular ions (Janev $2002-\mathrm{CH}_{4}^{+}, \mathrm{C}_{2} \mathrm{H}_{4}^{+}, \mathrm{C}_{2} \mathrm{H}_{6}^{+}, \mathrm{C}_{3} \mathrm{H}_{8}^{+}$; Derkatch, Minaev \& Larsson $2003-\mathrm{C}_{2} \mathrm{H}_{2}^{+}$), $\mathrm{CO}_{2}^{+}$(Seiersen et al. 2003), $\mathrm{NH}_{4}^{+}$(Ojekull et al. 2004), $\mathrm{N}_{2} \mathrm{H}^{+}$(Geppert et al. 2004a), and $\mathrm{SO}_{2}^{+}$(Geppert et al. 2004b).

\subsection{Ion-Atom and Atom-Atom Collisions}

Charge transfer plays an important role in a variety of environments and therefore has seen a substantial amount of activity over the report period. Studies for collisions on $\mathrm{H}$ include: $\mathrm{H}^{+}$(Cabrera-Trujullo et al. 2002a, Celiberto et al. 2002, Killian et al. 2004, Krstić et al. 2004), $\mathrm{He}^{2+}$ (Le et al. 2004), $\mathrm{Li}^{+-3+}$ (Purkait 2003), $\mathrm{Li}^{3+}$ and $\mathrm{Ne}^{10+}$ (Errea et al. 2004b), $\mathrm{B}^{2+}$ (Turner et al. 2003), $\mathrm{C}^{4+}$ (Liu et al. 2003), $\mathrm{C}^{4,5+}, \mathrm{N}^{5,6+}$, and $\mathrm{O}^{6,7+}$ (Kearns et al. 2003), $\mathrm{N}^{+}$(Lin et al. 2005), $\mathrm{N}^{2+}$ (Barragan et al. 2004), $\mathrm{N}^{4+}$ (CabreraTrujillo et al. 2002c), $\mathrm{N}^{5+}$ (Kearns et al. 2002), $\mathrm{O}^{+}$(Spirko et al. 2003), $\mathrm{O}^{2+}$ (Cabello et al. 2003), $\mathrm{O}^{3+}$ (Wang et al. 2003), $\mathrm{O}^{8+}$ and $\mathrm{Ar}^{8+}$ (Edgu-Fry et al. 2004, Lee et al. 2004), $\mathrm{Ne}^{2+}$ (Mroczkowski et al. 2003), $\mathrm{Ne}^{3+}$ (Rejoub et al. 2004), and $\mathrm{Ne}^{4+}$ (Havener et al. 2004). Collisions of neutral species with protons include: He* (Chibisov et al. 2002), N (Lin et al. 2005), Na (Watanabe et al. 2002, Le et al. 2003), S (Zhao et al. 2005a), Ar (Kirschner et al. 2004), and K (Watanabe et al. 2002, Hayakawa et al. 2004).

Neutral helium is also an important target for which studies have considered the incident ions: $\mathrm{H}^{+}$(Minami et al. 2004), Be ${ }^{3,4+}$ (Shimakura et al. 2003), $\mathrm{C}^{2+}$ (Gao \& Kwong 2003), $\mathrm{C}^{2-6+}, \mathrm{N}^{2-6+}, \mathrm{O}^{2-6+}$ (Ishii et al. 2004), $\mathrm{O}^{+}$(Lindsay \& Stebbings 2003, Zhao et al. 2005b), $\mathrm{O}^{5+}$ (Sobocinski et al. 2003), $\mathrm{O}^{6+}$ and $\mathrm{Ne}^{8+}$ (Bordenave-Montesquieu et al. 2003), $\mathrm{O}^{7+}$ (Shevelko et al. 2004), $\mathrm{Ne}^{2+}$ (Imai et al. 2003), $\mathrm{Ne}^{4+}$ (Kamber et al. 2003), $\mathrm{Ne}^{10+}$ (Rigazio et al. 2002, Ali et al. 2005), $\mathrm{S}^{2+}$ (Zhao et al. 2005c), $\mathrm{S}^{4+}$ (Wang et al. 2002), $\mathrm{Ar}^{9+}$ (Bliman et al. 2002), and $\mathrm{Fe}^{5-13+}$ (Cadế et al. 2003). The reverse process through radiative charge transfer has been considered for $\mathrm{O}+\mathrm{He}^{+}$(Zhao et al. 2004). Some relevant symmetric charge transfer studies where done for $\mathrm{N}^{+}+\mathrm{N}$ and $\mathrm{O}^{+}+$ O (Eletskii et al. 2004) On-line databases of charge transfer data include the National Institute for Fusion Science (NIFS) Charge Transfer Database Chart (2005) and the ORNL/UGA Charge Transfer Database for Astrophysics (2005) sites.

Elastic scattering and spin exchange have been studied for $\mathrm{H}^{+}+\mathrm{H}$ by Krstić et al. (2004) and Glassgold et al. (2005) while Zygelman et al. (2003) reported new spinexchange calculations for $\mathrm{H}+\mathrm{H}$. Chung \& Dalgarno (2002) studied diffusion of $\mathrm{H}$ in $\mathrm{He}$ and $\mathrm{He}$ in $\mathrm{H}$.

Molecules can be formed by collisions of atoms through radiative association. Bennett et al. (2003) studied the role of quasi-bound states in the formation of LiH. Atom collisions can also result in changes in internal energy and recent work includes: Li with $\mathrm{H}$ (Belyaev \& Barklem 2003), He* with He (Vrinceanu \& Sadeghpour 2002), and C and S with He (Le Picard et al. 2002).

\subsection{Ion-, Atom-, and Molecule-Molecule Collisions}

In photoionized environments, multiply charged ions may coexist with neutral molecules. Examples include x-ray ionized regions and solar wind interactions with cometary gas. In these environments charge transfer plays an important role. Recent studies of 
ion-molecule charge transfer include $\mathrm{H}^{+}$(Cabrera-Trujilla et al. 2002b, Savin et al. 2004), $\mathrm{H}^{+}$and $\mathrm{D}^{+}$(Kuskabe et al. 2004), $\mathrm{He}^{2+}$ (Errea et al. 2003), $\mathrm{C}^{2+}$ (Gao \& Kwong 2003), $\mathrm{C}^{4+}$ (Konnai et al. 2003), $\mathrm{C}^{4-5+}, \mathrm{N}^{5-6+}$, and $\mathrm{O}^{6-7+}$ (Kearns et al. 2003), $\mathrm{N}^{5+}$ (Kerns et al. 2002, Errea et al. 2004a), $\mathrm{O}^{+}$(Pichl et al. 2003), $\mathrm{O}^{3+}$ (Wang et al. 2004), $\mathrm{O}^{6+}$ (Bordenave-Montesquieu et al. 2003), $\mathrm{O}^{8+}$ and $\mathrm{Ar}^{8+}$ (Edgu-Fry et al. 2004), $\mathrm{S}^{2+}$ (Chen et al. 2003), and $\mathrm{Fe}^{3+}$ (Gao et al. 2003) with $\mathrm{H}_{2} ; \mathrm{C}^{2+}$ (Gao \& Kwong 2003), $\mathrm{C}^{3-4+}$ (Gao \& Kwong 2002), and $\mathrm{S}^{2+}$ (Chen et al. 2003) with CO; $\mathrm{H}^{+}$(Cabrera-Trujillo et al. 2002d), $\mathrm{C}^{2+}$ (Gao \& Kwong 2003), $\mathrm{S}^{2+}$ (Chen et al. 2003), and $\mathrm{Fe}^{3+}$ (Gae et al. 2003) with $\mathrm{N}_{2}$; $\mathrm{He}^{2+}$ with $\mathrm{H}_{2} \mathrm{O}$ and $\mathrm{CO}_{2}$ (Abu-Haija et al. 2003); and $\mathrm{H}^{+}$with $\mathrm{CH}_{2}$ (Suno et al. 2004).

For applications to x-ray emission from comets a number of investigations have considered a range of highly charged ions on various molecular targets including $\mathrm{Ne}^{+, 3,5,7,9+}$ with $\mathrm{H}_{2} \mathrm{O}$ (Pesic et al. 2004); $\mathrm{Ne}^{10+}$ with $\mathrm{H}_{2}, \mathrm{H}_{2} \mathrm{O}$, and $\mathrm{CO}_{2}$ (Rigazio et al. 2002); and $\mathrm{Ne}^{10+}$ with $\mathrm{CO}$ and $\mathrm{CO}_{2}$ (Ali et al. 2005). Also, see Krasnopolsky et al. (2004) for a review of cometary $\mathrm{x}$-ray relevant collision data.

In some environments, advances in modeling require vibrationally-resolved charge transfer and dissociation data. Such data are generally not available, but some studies have been performed for $\mathrm{H}^{+}+\mathrm{H}_{2}$ and $\mathrm{H}+\mathrm{H}_{2}^{+}$(Krstić \& Janev 2003), $\mathrm{O}^{3+}+\mathrm{H}_{2}$ (Wang et al. 2004), and $\mathrm{O}^{3+}+\mathrm{HD}$ (Stancil et al. 2004).

In low ionization environments, excited rovibrational states of molecules are formed through collisional excitation by atom and molecule impact. Investigations have been performed for excitation of $\mathrm{H}_{2}$ by $\mathrm{H}$ (Mielke et al. 2003), by $\mathrm{He}$ (Lee et al. 2005), by $\mathrm{H}_{2}$ (Ceballos et al. 2002, Lin \& Guo 2002), and by Li ${ }^{+}$(Roeggen et al. 2002); CO by H (Yang et al. 2005a), by He (Krems, et al. 2002, Carty et al. 2004, Smith et al. 2004, Yang et al. 2005b), and by $\mathrm{H}^{+}$(Kumar \& Kumar 2004); $\mathrm{N}_{2}$ by He (Stoecklin et al. 2002); $\mathrm{F}_{2}$ by $\mathrm{He}$ (Stoecklin et al. 2003); $\mathrm{SiO}$ by H (Palov et al. 2002); CaH by He (Krems et al. 2003); $\mathrm{H}_{2} \mathrm{O}$ by $\mathrm{H}_{2}$ (Dubernet \& Grosjean 2002, Grosjean et al. 2003, Weisenfeld et al. 2003); $\mathrm{N}_{2}$ by $\mathrm{N}_{2}$ (Ramos et al. 2002); $\mathrm{N}_{2}$ by CO (Cacciatore et al. 2004); CO by CO (Billings et al. 2003); and $\mathrm{CH}_{3} \mathrm{OH}$ by $\mathrm{He}$ (Flower and Davis 2004). A useful online database of rovibrational collisional excitation data, BASECOL, can be found at: http://amdpo.obspm.fr/basecol/.

Elastic scattering data for vibrationally-excited molecules have been presented by Krstić \& Schultz (2003) for $\mathrm{H}^{+}+\mathrm{H}_{2}$ and $\mathrm{H}+\mathrm{H}_{2}^{+}$collisions. An online database can be found at: http://cfadc.phy.ornl.gov/elastic/homeph2.html.

\subsection{Reactive Scattering and Chemistry}

Due to space limitations, we cannot review the many advances in reactive scattering and chemical processes relevant to astrophysics. However, some noteworthy and relevant studies include $\mathrm{OH}$ formation in $\mathrm{O}+\mathrm{H}_{2}$ collisions (Baunstein et al. 2004, Balakrishnan 2004, Sultanov \& Balakrishnan 2004), $\mathrm{CO}_{2}$ formation in $\mathrm{OH}+\mathrm{CO}$ collisions (Valero \& Kroes 2004), $\mathrm{OH}$ formation in $\mathrm{H}+\mathrm{H}_{2} \mathrm{O}$ collisions (Zhang et al. 2002), and $\mathrm{H}_{2}$ formation in $\mathrm{LiH}+\mathrm{H}$ collisions (Kim et al. 2003).

\section{Acknowledgements}

DRS acknowledges support from the DOE Office of Fusion Energy Sciences to Oak Ridge National Laboratory which is managed by UT-Battelle, LLC under contract No. DE-AC0500OR22725. The work of PCS was partially supported by NASA grant NAG5-11453. We thank Ms. Fay Ownby for assistance with preparing this manuscript.

**D.R. Schultz and P.C. Stancil** Chairpersons of the Working Group 


\section{References}

Abdellahi El Ghazaly, M. O. et al. 2004 J. Phys. B 37, 2467

Abu-Haija, O. et al. 2003, Nucl. Inst. Meth. B, 205, 634

Aggarwal, K. M. \& Keenan, F. P. 2002 Phys. Scr. 65, 383

Aggarwal, K. M. \& Keenan, F. P. 2003a Astron. Astrophys. 399, 799

Aggarwal, K. M. \& Keenan, F. P. 2003b Astron. Astrophys. 407, 769

Aggarwal, K. M., Keenan, F. P. \& Msezane, A. Z. 2003 Astron. Astrophys. 410, 349

Aggarwal, K. M. \& Keenan, F. P. 2004a Phys. Scr. 69, 385

Aggarwal, K. M. \& Keenan, F. P. 2004b Astron. Astrophys. 418, 371

Aggarwal, K. M. \& Keenan, F. P. 2005 Astron. Astrophys. 429, 1117

Aichele, K. et al. 2001 J. Phys. B 34, 4113

Akther, P. et al. 2002 J. Phys. B 35, L481

Al-Khalili, A. et al. 2003 Phys. Rev. A 68, 042702

Al-Mulla, S.Y.Y. \& Joensson, L. 2002 Phys. Scr. 65, 387

Al-Khalili, A. et al. 2003 Phys. Rev. A 68, 042702

Alfaz Uddin, M. \& Basak, A. K. 2003a Phys. Scr. 67, 37

Alfaz Uddin, M. \& Basak, A. K. 2003b Phys. Scr. 67, 112

Alfaz Uddin, M. et al. 2003 Phys. Scr. 68, 192

Ali, R. et al. 2005, Astrophys. J., 629, L125

Allan, M. \& Moreira, O. 2002 J. Phys. B 35, L37

Allan, M. 2002 J. Phys. B 35, L387

Allan, M. 2003 J. Phys. B 36, 2489

Allan, M. \& Skalicky, T. 2003 J. Phys. B 36, 3397

Allan, M. 2004 J. Phys. B 37, L359

Altun, Z. et al. 2004 Astron. Astrophys. 420, 775

Andersen, L. H., Herber, O. \& Zajfman,D. 2004 J. Phys. B 37, R57

Antony, B. K. et al. 2004 J. Phys. B 37, 1689

Ariyasinghe, W. M. \& Powers, D. 2002 Phys. Rev. A 66, 052716

Ariyasinghe, W. M., Wijerathna, T. \& Powers, D. 2003 Phys. Rev. A 68, 032708

Ariyasinghe, W. M. et al. 2004 Nucl. Inst. Meth. B 217, 389

Ariyasinghe, W. M. \& Goains, C. 2004 Phys. Rev. A 70, 052709

Badnell, N. R. et al. 2003 Astron. Astrophys. 406, 1151

Badnell, N. R. et al. 2004 J. Phys. B 37, 4589

Baek, W. Y. \& Grosswendt, B. 2003 J. Phys. B 36, 731

Bahati, E. M. et al. 2001a J. Phys. B 34, 1757

Bahati, E. M. et al. 2001b J. Phys. B 34, 2963

Balakrishnan, N. 2004, J. Chem. Phys., 121, 6346

Ballance, C. P., Badnell, N. R. \& Berrington, K. A. 2002 J. Phys. B 35, 1095

Ballance, C. P. et al. 2003a J. Phys. B 36, 235

Ballance, C. P. et al. 2003b Phys. Rev. A 68, 062705

Ballance, C. P. \& Griffin, D. C. 2004 J. Phys. B 37, 2943

Baluja, K. L. \& Msezane, A. Z. 2001 J. Phys. B 34, 3057

Baluja, K. L. \& Msezane, A. Z. 2002 J. Phys. B 35, 437

Bannister, M. E. et al. 2002 Phys. Rev. A 66, 032707

Bannister, M. E. et al. 2003 Phys. Rev. A 68, 042714

Barragan, P. 2004, Phys. Rev. A, 70, 022707

Bartlett, P. L. \& Stelbovics, A. T. 2002 Phys. Rev. A 66, 012707

Bartlett, P. L., Stelbovics, A. T. \& Bray, I. 2003 Phys. Rev. A 68, 030701(R)

Bartlett, P. L. \& Stelbovics, A. T. 2004 Phys. Rev. A 69, 040701 
Bartschat, K. \& Sadeghpour, H. R. 2003 J. Phys. B 36, L9

Bartschat, K. \& Andersen, N. 2003 J. Phys. B 36, 163

Bartschat, K. et al. 2004 J. Phys. B 37, 2617

Bartschat, K., Dasgupta, A. \& Madison, D. H. 2004 Phys. Rev. A 69, 062706

Basner, R., Schmidt, M. \& Becker, K. 2003 J. Chem. Phys. 118, 2153

Baunstein, M. et al. 2004, J. Chem. Phys., 120, 4316

Bautista, M. A. 2003 J. Phys. B 36, 1503

Bautista, M. A. et al. 2003 Astron. Astrophys. 403, 339

Bautista, M. A. et al. 2004 Astron. Astrophys. 418, 1171

Bautista, M. A. 2004 Astron. Astrophys. 420, 763

Becker, C. et al. 2004 J. Phys. B 37, 1503

Behar, E. et al. 2004 Phys. Rev. A 69, 022704

Beigman, I., Defrance, P. \& Vainshtein, L. 2003a J. Phys. B 36, 2019

Beigman, I., Defrance, P. \& Vainshtein, L. 2003b Nucl. Inst. Meth. B 205, 427

Belyeav, A. K. \& Barklem, P. S. 2003, Phys. Rev. A, 68, 062703

Bennett, O. J. et al. 2003, Mon. Not. Roy. Astron. Soc., 341, 361

Berrington, K. A. \& Ballance, C. P. 2002 J. Phys. B 35, 2275

Bettega, M.H.F. \& Lima, M.A.P. 2004 J. Phys. B 37, 3859

Bettega, M.H.F., Lima, M.A.P. \& Ferreira, L. G. 2004 Phys. Rev. A 70, 062711

Bhatia, A. K. 2002 Phys. Rev. A 66, 064702

Bhatia, A. K. \& Landi, E. 2003 Astrophys. J., Pt. 1 585, 587

Biava, D. A. et al. 2002 J. Phys. B 35, 293

Billings, G. D. 2003, J. Phys. B., 36, 1175

Blanco, F. \& Garcia, G. 2003 Phys. Rev. A 67, 022701

Bliman, S. et al. 2002, Phys. Rev. A, 66, 052707

Boechat-Roberty, H. M. et al. 2002 J. Phys. B 35, 1409

Boehm, S. et al. 2003 Astron. Astrophys. 405, 1157

Boffard, J. B. et al. 2001 Phys. Rev. A 64, 032708

Bordenave-Montesquieu, D. et al. 2003, J. Phys. B, 36, 65

Bray, I., Fursa, D. V. \& Stelbovics, A. T. 2003 J. Phys. B 36, 2211

Brescansin, L. M. et al. 2004 J. Phys. B 37, 471

Brown, G. V., Beiersdorfer, P. \& Widmann, K. 2001 Phys. Rev. A 63, 032719

Bureyeva, L. A. et al. 2002a J. Phys. B 35, 2505

Bureyeva, L. A. et al. 2002b Phys. Rev. A 65, 032702

Burke, P. G. et al. 2002 Phys. Scr. T100, 55

Cabello, C. M. et al. 2003, J. Phys. B, 36, 307

Cabrera-Trujillo, R. et al. 2002a, J. Chem. Phys., 116, 2783

Cabrera-Trujillo, R. et al. 2002b, Phys. Rev. A, 65, 024901

Cabrera-Trujillo, R. et al. 2002c, Phys. Rev. A, 66, 022706

Cabrera-Trujillo, R. et al. 2002d, Phys. Rev. A, 66, 042712

Cacciatore, M. et al. 2004, J. Phys. B, 37, 3379

Cadế, I. et al. 2003, J. Phys. B, 36, 3303

Carty, D. et al. 2004, J. Chem. Phys., 121, 4671

Ceballos, A. et al. 2002, J. Phys. Chem. Ref. Data, 31, 371

Celiberto, R., Capitelli, M. \& Laricchiuta, A. 2002 Phys. Scr. T96, 32

CFADC 2002, www-cfadc.phy.ornl.gov

Champion, C., Hanssen, J. \& Hervieux, P. A. 2002a J. Chem. Phys. 117, 197

Champion, C., Hanssen, J. \& Hervieux, P. A. 2002 b Phys. Rev. A 65, 022710

Champion, C., Hanssen, J. \& Hervieux, P.-A. 2004 J. Chem. Phys. 121, 9423

Chang, J.-C. et al. 2004 Phys. Rev. A 69, 052713 
Chen, D. et al. 2003, Phys. Rev. A, 68, 052703

Chen, G.-X., Pradhan, A. K. \& Eissner, W. 2003 J. Phys. B 36, 453

Chen, M. H. 2002 Phys. Rev. A 66, 052715

Chen, Z. et al. 2004 J. Phys. B 37, 981

Chibisov, M. I. et al. 2002, J. Phys. B, 35, 5081

Chidichimo, M. C., Badnell, N. R. \& Tully, J. A. 2003 Astron. Astrophys. 401, 1177

Childers, J. G. et al. 2003 Phys. Rev. A 68, 030702(R)

Childers, J. G. et al. 2004 Phys. Rev. A 69, 022709

Cho, H. et al. 2004a J. Phys. B 37, 625

Cho, H. et al. 2004b J. Phys. B 37, 4639

Chung, H.-K. \& Dalgarno, A. 2002, Phys. Rev. A, 66, 012712

CIAMDA 98, 1998, Computer Index to Atomic and Molecular Collision Data (Vienna:

International Atomic Energy Agency)

Colgan, J., Pindzola, M. S. \& Robicheaux, F. 2002 Phys. Rev. A 66, 012718

Colgan, J. et al. 2003a Astron. Astrophys. 412, 597

Colgan, J. et al. 2003b Phys. Rev. A 68, 032712

Colgan, J., Pindzola, M. S. \& Badnell, N. R. 2004 Astron. Astrophys. 417, 1183

Cooper, J. W. \& van Boeyen, R. W. 2004 J. Phys. B 37, L77

Curik, R. \& Gianturco, F. A. 2002 J. Phys. B 35, 717

Curik, R. \& Carsky, P. 2003 J. Phys. B 36, 2165

da Costa, R. F., da Paixao, F. J. \& Lima, M.A.P. 2004 J. Phys. B 37, L129

Dasgupta, A. \& Whitney, K. G. 2004 Phys. Rev. A 69, 022702

Date, H., Ishimaru, Y. \& Shimozuma, M. 2003 Nucl. Inst. Meth. B 207, 373

de Pablos, J. L. et al. 2002 J. Phys. B 35, 865

Deb, N. C. \& Crothers, D.S.F. 2002 Phys. Rev. A 65, 052721

Defrance, P. et al. 2003 Nucl. Inst. Meth. B 205, 405

Delahaye, F. \& Pradhan, A. K. 2002 J. Phys. B 35, 3377

Demesie, A. M. et al. 2003 J. Phys. B 36, 665

Denifl, S. et al. 2002 J. Phys. B 35, 4685

Derkatch, A. M., Minaev, B. \& Larsson, M. 2003 Phys. Scr. 67, 407

Deutsch, H. et al. 2002 J. Phys. B 35, L65

Dogan, M. \& Crowe, A. 2002 J. Phys. B 35, 2773

Dubernet, M.-L. \& Grosjean, A. 2002, Astron. Astrophys., 390, 793

Dunn, G. H. et al. 2002 Phys. Rev. A 66, 032706

Edgu-Fry, E. et al. 2004, Phys. Rev. A, 69, 052714

Eletskii, A. V. et al. 2004, Phys. Rev. A, 69, 042718

Errea, L. F. et al. 2003, J. Phys. B, 36, L135

Errea, L. F. et al. 2004a, Phys. Rev. A, 69, 012705

Errea, L. F. et al. 2004b, Phys. Rev. A, 70, 052713

Erwin, D. A. \& Kunc, J. A. 2003 J. Phys. B 36, 4605

Fabrikant, I. I., Wadehra, J. M. \& Xu, Y. 2002 Phys. Scr. T96, 45

Faure, A. \& Tennyson, J. 2002a J. Phys. B 35, 1865

Faure, A. \& Tennyson, J. 2002b J. Phys. B 35, 3945

Faure, A., Gorfinkiel, J. D. \& Tennyson, J. 2004 J. Phys. B 37, 801

Feng, H., Sun, W. \& Morrison, M. A. 2003 Phys. Rev. A 68, 062709

Florescu, A. I. et al. 2003 J. Phys. B 36, 1205

Flower, D. R. \& Davis, S. L. 2004, J. Phys. B, 37, 165

Fogle, M. et al. 2003a Astron. Astrophys. 409, 781

Fogle, M. et al. 2003b J. Phys. B 36, 2563

Fritioff, K. et al. 2003 Euro. Phys. J: D 27, 23 
Fritioff, K. et al. 2004 Phys. Rev. A 69, 042707

Fursa, D. V. \& Bray, I. 2001 Phys. Rev. A 63, 032708

Fursa, D. V. \& Bray, I. 2003 J. Phys. B 36, 1663

Gao, K. et al. 2003, Phys. Rev. A, 67, 022702

Gao, H. \& Kwong, V. H. S. 2003, Phys. Rev. A, 68, 052704

Gao, H. \& Kwong, V. H. S. 2004, Phys. Rev. A, 69, 052715

Garcia, G., Blanco, F. \& Williart, A. 2001 Chem. Phys. Lett. 335, 227

Garcia, G. et al. 2002 J. Phys. B 35, 4657

Geppert, W. D. et al. 2004a Astrophys. J., Pt. 1 609, 459

Geppert, W. D. et al. 2004b Astrophys. J., Pt. 1 610, 1228

Ghosh, D. \& Sinha, C. 2003 J. Phys. B 36, 675

Ghosh, D., Nath, B. \& Sinha, C. 2003 J. Phys. B 36, 1479

Gianturco, F. A. \& Stoecklin, T. 2001 J. Phys. B 34, 1695

Glassgold, A. E. et al. 2005, Astrophys. J., 621, 808

Glosik, J. et al. 2001 J. Phys. B 34, L485

Gorczyca, T. W., Badnell, N. R. \& Savin, D. W. 2002 Phys. Rev. A 65, 062707

Gorfinkiel, J. D. \& Tennyson, J. 2004 J. Phys. B 37, L343

Green, M. A. et al. 2002a J. Phys. B 35, 567

Green, M. A. et al. 2002b J. Phys. B 35, 3793

Griffin, D. C. et al. 2001 Phys. Rev. A 64, 032718

Grosjean, A. et al. 2003, Astron. Astrophys., 408, 1197

Grujic, P. V. 2003 Phys. Scr. 68, C90

Gstir, B. et al. 2002 J. Phys. B 35, 2993

Gstir, B. et al. 2003 Nucl. Inst. Meth. B 205, 413

Gu, M. F. 2003a Astrophys. J., Pt. 1 589, 1085

Gu, M. F. 2003b Astrophys. J., Pt. 1 590, 1131

Gu, M. F. 2003c Astrophys. J., Pt. 1 593, 1249

Gu, M. F. 2004 Phys. Rev. A 70, 062704

Gupta, G. P. \& Msezane, A. Z. 2002 Phys. Scr. 66, 354

Hanel, G. et al. 2002 J. Chem. Phys. 116, 2456

Harb, T., Kedzierski, W. \& McConkey, J. W. 2000 J. Chem. Phys. 115, 5507

Harries, J. R., Hammond, P. \& Murray, A. J. 2003 J. Phys. B 36, 2579

Havener, C. C. et al. 2004, Phys. Rev. A, 71, 034702

Haxton, D. J. et al. 2004 Phys. Rev. A 69, 062714

Hayakawa, S. et al. 2004, Phys. Rev. A, 70, 022708

Heerlein, C., Zwicknagel, G. \& Toepffer, C. 2002 Phys. Rev. Lett. 89, 083202

Horacek, J. et al. 2004 Phys. Rev. A 70, 052712

Houamer, S. et al. 2003 J. Phys. B 36, 3009

Huang, M.-T. et al. 2002 Phys. Rev. A 66, 012715

Hudson, J. E., Vallance, C. \& Harland, P. W. 2004 J. Phys. B 37, 445

Imai, T. W. et al. 2003, Phys. Rev. A, 68, 012716

Isaacs, W. A. et al. 2001 Phys. Rev. A 63, 030704

Ishii, K. et al. 2004, Phys. Rev. A, 70, 042716

Itikawa, Y. 2004 J. Phys. B 37, R1

Jablonski, A., Salvat, F. \& Powell, C. J. 2004 J. Phys. Chem. Ref. Data 33, 409 James, G. K. et al. 2002 Phys. Rev. A 66, 042710

James, K. E., Childers, J. G. \& Khakoo, M. A. 2004 Phys. Rev. A 69, 022710

Janev, R. K. 2002 Phys. Scr. T96, 94

Janev, R. K. \& Reiter, D. 2003 J. Nucl. Mater. 313-316, 1202

Janzen, P. H. et al. 2003 Phys. Rev. A 67, 052702 
Jha, L. K. 2002 Phys. Scr. 66, 228

Jha, L. K. \& Roy, B. N. 2002 Euro. Phys. J: D 20, 5

Jiao, C. Q., DeJoseph, C. A. \& Garscadden, A. 2002 J. Chem. Phys. 117, 161

Johnson, P. V. \& Kanik, I. 2001 J. Phys. B 34, 3041

Johnson, P. V. et al. 2003a J. Phys. B 36, 3203

Johnson, P. V. et al. 2003b J. Phys. B 36, 4289

Joshipura, K. N., Antony, B. K. \& Vinodkumar, M. 2002 J. Phys. B 35, 4211

Joshipura, K. N. et al. 2004 Phys. Rev. A 69, 022705

Kai, T. et al. 2004 J. Phys. Soc. Japan 73, 885

Kai, T., Srivastava, R. \& Nakazaki, S. 2004 Phys. Rev. A 70, 062705

Kalhori, S. et al. 2004 Phys. Rev. A 69, 022713

Kamber, E. Y. et al. 2003, Nucl. Inst. Meth. B, 205, 577

Kampp, M. et al. 2002 J. Phys. B 35, 2325

Kanik, I., Johnson, P. V. \& James, G. K. 2001 J. Phys. B 34, 1685

Kearns, D. M. et al. 2002, J. Phys. B. 35, 4335

Kearns, D. M. et al. 2003, J. Phys. B. 36, 3653

Kedzierski, W. 2002 J. Phys. B 35, 4401

Keller, S. 2003 J. Phys. B 36, 755

Khakoo, M. A. et al. 2002 Phys. Rev. A 65, 062711

Kheifets, A. S. 2004 Phys. Rev. A 69, 032712

Kheifets, A. S. \& Bray, I. 2004 Phys. Rev. A 69, 050701

Khouilid, M. et al. 2001 J. Phys. B 34, 1727

Killian, B. J. et al. 2004, J. Phys. B, 37, 4733

Kim, K. H. et al. 2003 J. Chem. Phys., 119, 4689

Kim, Y.-K. 2001 Phys. Rev. A 64, 032713

Kim, Y.-K. \& Desclaux, J.-P. 2002 Phys. Rev. A 66, 0127080

Kim, Y.-K. 2002 Phys. Rev. A 65, 022705

Kimura, M., Makochekanwa, C. \& Sueoka, O. 2004 J. Phys. B 37, 1461

Kirschner, T. et al. 2004, Phys. Rev. A, 69, 012708

Kisielius, R. \& Storey, P. J. 2002 Astron. Astrophys. 387, 1135

Kobayashi, A. et al. 2002 J. Phys. B 35, 2087

Kokoouline, V. \& Greene, C. H. 2003a Phys. Rev. A 68, 012703

Kokoouline, V. \& Greene, C. H. 2003b Phys. Rev. Lett. 90, 133201

Konnai, A. et al. 2003, Nucl. Inst. Meth. B, 205, 586

Krasheninnikov, S. I. 2002 Phys. Scr. T96, 7

Krasnopolsky, V. et al. 2004, Space Sci. Reviews, 113, 271

Krems, R. V. et al. 2002, J. Chem. Phys., 116, 4517

Krems, R. V. et al. 2003, Phys. Rev. A, 67, 060703

Kroin, T., Michelin, S. E. \& Lee, M.-T. 2001 J. Phys. B 34, 1829

Kroin, T. et al. 2003 Phys. Rev. A 68, 012701

Krstić, P. S. 2002, Phys. Rev. A, 66, 042717

Krstić, P. S. \& Schultz, D. R. 2003, J. Phys. B, 36, 385

Krstić, P. S. \& Janev, R. K. 2003, Phys. Rev. A, 67, 022708

Krstić, P. S. 2004, J. Phys. B, 37, L217

Krstić, P. S. et al. 2004, Phys. Rev. A, 70, 042711

Kumar, T. J. D. \& Kumar, S. 2004, J. Chem. Phys., 121, 191

Kuo, T.-Y. \& Huang, K.-N. 2001 Phys. Rev. A 64, 032710

Kusakabe, T. et al. 2004, Phys. Rev. A, 70, 052710

Landi, E. \& Bhatia, A. K. 2003 Astrophys. J., Pt. 1 589, 1075

Landi, E., Storey, P. J. \& Zeippen, C. J. 2004 Astrophys. J., Pt. 1 607, 640 
Laricchiuta, A., Celiberto, R. \& Janev, R. K. 2004 Phys. Rev. A 69, 022706

Le, A.-T. et al. 2003, Phys. Rev. A, 68, 012705

Le, A.-T. et al. 2004, Phys. Rev. A, 69, 062703

Le Padellec, A. et al. 2002 J. Phys. B 35, 3669

Le Picard, S. D. et al. 2002, J. Chem. Phys., 117, 10109

Lee, M.-T. et al. 2002a Phys. Rev. A 65, 062702

Lee, M.-T. et al. 2002b Phys. Rev. A 66, 062703

Lee, M.-T. \& Mazon, K. T. 2002 Phys. Rev. A 65, 042720

Lee, T.-G. et al. 2004, Phys. Rev. A, 70, 012702

Lee, T.-G. et al. 2005, J. Chem. Phys., 122, 024307

Lepp, S., Stancil, P. C. \& Dalgarno, A. 2002 J. Phys. B 35, R57

Li, W.-B. et al. 2003 Phys. Rev. A 67, 062708

Lin, C.-Y. et al. 2005, Phys. Rev. A, 71, 062708

Lin, S. Y. \& Guo, H. 2002, J. Chem. Phys., 117, 5183

Lindsay, B. G., Rejoub, R. \& Stebbings, R. F. 2003 J. Chem. Phys. 118, 5894

Lindsay, B. G. \& Stebbings, R. F. 2003, Phys. Rev. A, 67, 022715

Linert, I., Zubek, M. \& Zubek, M. 2004 J. Phys. B 37, 4681

Liu, C.-N. et al. 2003, Phys. Rev. A, 68, 062702

Liu, X. et al. 2003 J. Phys. B 36, 173

Liu, X. \& Shemansky, D. E. 2004 Astrophys. J., Pt. 1 614, 1132

Llovet, X., Merlet, C. \& Salvat, F. 2002 J. Phys. B 35, 973

Loch, S. D. et al. 2002 Phys. Rev. A 66, 052708

Loch, S. D. et al. 2003 Phys. Rev. A 67, 042714

Lopes, A. R. \& Bettega, M.H.F. 2003 Phys. Rev. A 67, 032711

Lopes, A. R. et al. 2004 Phys. Rev. A 69, 014702

Luna, H. et al. 2003 J. Phys. B 36, 4717

Machado, A. M. et al. 2001 Phys. Rev. A 63, 032707

Madison, D. H. et al. 2004 J. Phys. B 37, 1073

Madzunkov, S. et al. 2002 Phys. Rev. A 65, 032505

Maia, C.A.S. \& Bettega, M.H.F. 2003 Phys. Rev. A 67, 042710

Makochekanwa, C., Sueoka, O. \& Kimura, M. 2003 Phys. Rev. A 68, 032707

Makochekanwa, C., Sueoka, O. \& Kimura, M. 2004 J. Phys. B 37, 1841

Mandy, M. E. et al. 2004, J. Chem. Phys., 120, 5585

Mansouri, A. et al. 2004 J. Phys. B 37, 1203

Maté, B. et al. 2005, J. Chem. Phys., 122, 064313

McCall, B. J. et al. 2004 Phys. Rev. A 70, 052716

McCurdy, C. W. et al. 2003 Phys. Rev. A 67, 042708

McEachran, R. P. \& Stauffer, A. D. 2003 J. Phys. B 36, 3977

McLaughlin, B. M., Daw, A. \& Bell, K. L. 2002 J. Phys. B 35, 283

McLaughlin, B. M. et al. 2002 J. Phys. B 35, 2755

Meneses, G. D. et al. 2002 J. Phys. B 35, 3119

Merabet, H. et al. 2003a J. Phys. B 36, 3383

Merabet, H. et al. 2003b Nucl. Inst. Meth. B 205, 399

Merz, R. \& Linder, F. 2003a J. Phys. B 36, 1143

Merz, R. \& Linder, F. 2003b J. Phys. B 36, 2921

Michelin, S. E. et al. 2003 J. Phys. B 36, 1525

Mielke, S. L. 2003, Phys. Rev. Lett., 91, 063201

Milosavljevic, A. R., Sevic, D. \& Marinkovic, B. P. 2004 J. Phys. B 37, 4861

Minami, T. et al. 2004, J. Phys. B, 37, 4025

Mitnik, D. M. et al. 2003 J. Phys. B 36, 717 
Mitnik, D. M. \& Badnell, N. R. 2004 Astron. Astrophys. 425, 1153

Mityureva, A. A., Smirnov, V. V. \& Ponomarenko, G. A. 2002 Opt. Spectrosc. 92, 325

Mohamed, T. et al. 2002 Phys. Rev. A 66, 022719

Mroczkowski, T. et al. 2003, Phys. Rev. A, 68, 032721

Muktavat, K., Srivastava, R. \& Stauffer, A. D. 2002 J. Phys. B 35, 4797

Murakami, I., Safronova, U. I. \& Kato, T. 2002 Can. J. Phys. 80, 1525

Nakano, Y. et al. 2002 Phys. Rev. A 66, 032714

National Institute for Fusion Science (NIFS) Charge Transfer Database Chart (2005); amdata.nifs.ac.jp

Neau, A. et al. 2002 Phys. Rev. A 65, 044701

Ngassam, V. et al. 2003 Phys. Rev. A 68, 032704

Niimura, M., Smith, S. J. \& Chutjian, A. 2002 Astrophys. J., Pt. 1 565, 645

Nikolic, D. et al. 2004 Phys. Rev. A 70, 062723

Nishimura, T. \& Gianturco, F. A. 2002 J. Phys. B 35, 2873

O'Rourke, B. E. et al. 2003 Nucl. Inst. Meth. B 205, 378

O'Rourke, B. E. et al. 2004 J. Phys. B 37, 2343

Ojekull, J. et al. 2004 J. Chem. Phys. 120, 7391

ORNL/UGA Charge Transfer Database for Astrophysics (2005); cfadc.phy .ornl. gov/astro/ps/data/

Palov, A. P. et al. 2002, J. Chem. Phys., 116, 1388

Panajotovic, R. et al. 2003 J. Phys. B 36, 1615

Papp, Z. \& Hu, C.-Y. 2002 Phys. Rev. A 66, 052714

Pesic, Z. D. et al. 2004, J. Phys. B, 37, 1405

Pichl, L. et al. 2003, J. Chem. Phys., 118, 4872

Pigarov, A. Yu. 2002 Phys. Scr. T96, 16

Pindzola, M. S. 2002 Phys. Rev. A 65, 014701

Pindzola, M. S. et al. 2004 Phys. Rev. A 70, 032705

Plummer, M., Noble, C. J. \& Le Dourneuf, M. 2004 J. Phys. B 37, 2979

Poparic, G., Vicic, M. \& Belic, D. S. 2001 J. Phys. B 34, 381

Poparic, G. B., Vicic, M. D. \& Belic, D. S. 2002 Phys. Rev. A 66, 022711

Popovic, D. B. et al. 2002 Phys. Rev. A 65, 034704

Purkait, M. et al. 2003, Nucl. Inst. Meth. B, 207, 101

Qi, J. B. et al. 2002a J. Phys. B 35, 829

Qi, J. B. et al. 2002b Phys. Rev. A 65, 032720

Ramos, A. et al. 2002, Phys. Rev. A, 66, 022702

Ramsbottom, C. A. et al. 2002 J. Phys. B 35, 3451

Ramsbottom, C. A. et al. 2004 J. Phys. B 37, 3609

Rejoub, R., Lindsay, B. G. \& Stebbings, R. F. 2002 Phys. Rev. A 65, 042713

Rejoub, R. et al. 2004, Phys. Rev. A, 69, 052704

Rescigno, T. N. et al. 2002 Phys. Rev. A 65, 032716

Rigazio, M. et al. 2002, Phys. Rev. A, 66, 064701

Rocha, A. B. \& Bielschowsky, C. E. 2002 Phys. Rev. A 66, 052720

Roeggen, I. et al. 2002, J. Phys. B, 35, 1707

Royal, J., Larson, A. \& Orel, A. E. 2004 J. Phys. B 37, 3075

Salvat, F. 2003 Phys. Rev. A 68, 012708

Santos, J. P., Parente, F. \& Kim, Y.-K. 2003 J. Phys. B 36, 4211

Savin, D. W. et al. 2002 Astrophys. J., Pt. 1 576, 1098

Savin, D. W. et al. 2004, Astrophys. J., 606, L157; 607, L147

Schappe, R. S., Edgell, R. J. \& Urban, E. 2002 Phys. Rev. A 65, 042701

Schippers, S. et al. 2004 Astron. Astrophys. 421, 1185 
Schnell, M. et al. 2003 Nucl. Inst. Meth. B 205, 367

Schuch, R. 2003 Phys. Scr. T104, 181

Schultz, D. R., \& Stancil, P. C. 2002 Reports on Astronomy XXVA, 1001-2002

Seiersen, K. et al. 2003 Phys. Rev. A 68, 022708

Serov, V. V. et al. 2002 Phys. Rev. A 65, 062708

Shevelko, V. P. et al. 2004, J. Phys. B, 37, 201

Shimakura, N. et al. 2003, Nucl. Inst. Meth. B, 205, 596

Sienkiewicz, J. E. et al. 2002 Phys. Lett. A, 293, 183

Skalicky, T. \& Allan, M. 2004 J. Phys. B 37, 4849

Smith, A.C.H. et al. 2003 Nucl. Inst. Meth. B 205, 421

Smith, S. J. et al. 2003 Phys. Rev. A 68, 062708

Smith, T. C. et al. 2004, J. Chem. Phys., 120, 2285

Sobocinski, P. et al. 2003, J. Phys. B, 36, 1283

Sobrinho, A.M.C., Lozano, N.B.H. \& Lee, M.-T. 2004 Phys. Rev. A 70, 032717

Sorokin, A. A. et al. 2004 J. Phys. B 37, 3215

Spinko, J. A. et al. 2003, J. Phys. B, 36, 1645

Srivastava, R., McEachran, R. P. \& Stauffer, A. D. 2002 Can. J. Phys. 80, 687

Stancil, P. C. et al. 2004, Faraday Discuss., 127, 73

Stano, M. et al. 2003 J. Phys. B 36, 261

Starobinets, A. et al. 2003 Phys. Scr. 67, 500

Stewart, M. D. et al. 2002 Phys. Rev. A 65, 032704

Stoecklin, T. et al. 2002, Phys. Rev. A, 66, 042703

Stoecklin, T. et al. 2003, Phys. Rev. A, 68, 032716

Storey, P. J., Zeippen, C. J. \& Le Dourneuf, M. 2002 Astron. Astrophys. 394, 753

Strasser, D. et al. 2002a Phys. Rev. A 65, 010702

Strasser, D. et al. 2002b Phys. Rev. A 66, 032719

Sultanov, R. A. \& Balakrishnan, N. 2004, J. Chem. Phys., 121, 11038

Suno, H. et al. 2004, Phys. Rev. A, 70, 032703

Sweeney, C. J., Grafe, A. \& Shyn, T. W. 2001 Phys. Rev. A 64, 032704

Sweeney, C. J., Grafe, A. \& Shyn, T. W. 2004 Phys. Rev. A 69, 052709

Szluniska, M., Van Reeth, P. \& Laricchia, G. 2002 J. Phys. B 35, 4059

Szmytkowski, C. \& Kwitnewski, S. 2003 J. Phys. B 36, 2129

Szmytkowski, C., Kwitnewski, S. \& Ptasinska-Denga, E. 2003 Phys. Rev. A 68, 032715

Szmytkowski, C. et al. 2004 J. Phys. B 37, 1833

Takagi, H. 2002 Phys. Scr. T96, 52

Takagi, H. 2004 Phys. Rev. A 70, 022709

Takahashi, M. et al. 2003 J. Phys. B 36, 2539

Tayal, S. S. 2002 Phys. Rev. A 66, 030701

Terrell, C. A., Hansen, D. L. \& Ajello, J. M. 2004 J. Phys. B 37, 1931

Thomas, R. et al. 2002 Phys. Rev. A 66, 032715

Trevisan, C. S. \& Tennyson, J. 2001 J. Phys. B 34, 2935

Trevisan, C. S., Orel, A. E. \& Rescigno, T. N. 2003 Phys. Rev. A 68, 062707

Tsurubuchi, S., Kobayashi, H. \& Hyodo, M. 2003 J. Phys. B 36, 2629

Turner, A. R. et al. 2003, Phys. Rev. A, 68, 012704

Uddin, M. A. et al. 2004a J. Phys. B 37, 1909

Uddin, M. A. et al. 2004b Phys. Rev. A 70, 032706

Valero, R. \& Kroes, G.-J. 2004, Phys. Rev. A, 70, 0407011

van Zoest, T. et al. 2004 J. Phys. B 37, 4387

Vanroose, W. et al. 2004 Phys. Rev. Lett. 92, 053201

Vinodkumar, M. et al. 2003 Nucl. Inst. Meth. B 212, 63 
Vrinceanu, D. \& Sadeghpour, H. R. 2002, Phys. Rev. A, 65, 062712

Wang, J. G. et al. 2002, J. Phys. B, 35, 3137

Wang, J. G. et al. 2003, Phys. Rev. A, 67, 012710

Wang, J. G. et al. 2004, Phys. Rev. A, 69, 062702

Watanabe, A. et al. 2002, Phys. Rev. A, 66, 044701

Watanabe, H. et al. 2003 Nucl. Inst. Meth. B 205, 417

Weber, T., Boffard, J. B. \& Lin, C. C. 2003 Phys. Rev. A 68, 032719

Whiteford, A. D. et al. 2001 J. Phys. B 34, 3179

Whiteford, A. D. et al. 2002 J. Phys. B 35, 3729

Wiesenfeld, L. et al. 2003, J. Phys. B, 36, 1319

Witthoeft, M. C., Pindzola, M. S. \& Colgan, J. 2003 Phys. Rev. A 67, 032713

Witthoeft, M. et al. 2003 Phys. Rev. A 68, 022711

Witthoeft, M. C., Loch, S. D. \& Pindzola, M. S. 2004 Phys. Rev. A 70, 022711

Woeste, G. et al. 2002 J. Phys. B 35, 4847

Wrkich, J. et al. 2002 J. Phys. B 35, 4695

Yan, J., Li, Y.-M. \& Yao, J.-H. 2003 Nucl. Inst. Meth. B 205, 382

Yang, B.H. et al. 2005a, J. Chem. Phys, 123, 094308

Yang, B.H. et al. 2005b, J. Chem. Phys, 123, 134326

Yu, D. H. et al. 2003 Phys. Rev. A 67, 032707

Yuan, Z.-S. et al. 2002 Phys. Rev. A 66, 062701

Zatsarinny, O. \& Tayal, S. S. 2002a J. Phys. B 35, 241

Zatsarinny, O. \& Tayal, S. S. 2002b J. Phys. B 35, 2493

Zatsarinny, O. \& Fischer, C. F. 2002 J. Phys. B 35, 4669

Zatsarinny, O. et al. 2003 Astron. Astrophys. 412, 587

Zatsarinny, O. et al. 2004a Astron. Astrophys. 417, 1173

Zatsarinny, O. et al. 2004b Astron. Astrophys. 426, 699

Zatsarinny, O. \& Bartschat, K. 2004a J. Phys. B 37, 2173

Zatsarinny, O. \& Bartschat, K. 2004b J. Phys. B 37, 4693

Zhang, D. H. et al. 2002, Phys. Rev. Lett., 89, 283293

Zhang, H. L., Nahar, S. N. \& Pradhan, A. K. 2001 Phys. Rev. A 64, 032719

Zhang, H. L. et al. 2002 J. Phys. B 35, 3829

Zhang, H. L. \& Sampson, D. H. 2002 Phys. Rev. A 66, 042704

Zhang, Z. et al. 2004 Phys. Rev. A 69, 062711

Zhao, L. B. et al. 2004, Astrophys. J., 615, 1063

Zhao, L. B. et al. 2005a, Phys. Rev. A, 71, R060701

Zhao, L. B. et al. 2005b, Phys. Rev. A, 71, 062713

Zhao, L. B. et al. 2005c, Phys. Rev. A, 72, 032719

Zygelman, B. et al. 2003, Phys. Rev. A, 67, 042715

\section{Working Group on Line Broadening PRESIDENTS: N.F. Allard and G. Peach}

Two conferences held in 2004 and 2005 have been devoted to the subject of Spectral Line Shapes. The 17th International Conference on Spectral Line Shapes (ICSLS) was held at the Université Pierre et Marie Curie in Paris (France) in 2004; the proceedings have been published by the Editions Frontier group and include a special section on astrophysical applications. The 5th Serbian Conference on Spectral Line Shapes in 
Astrophysics (SCSLSA) was held in Vršac in 2005 and the proceedings have been published in Memorie della Supplementi degli Società Astronomica Italiana (Mem. S.A.It.).

In the following report, we select experimental and theoretical work that may be of interest for astrophysics.

\subsection{Stark broadening}

The Critical Review of Selected Data on Experimental Stark Widths and Shifts for Spectral Lines of Neutral and Ionized Atoms for the period 1989-2000, see Konjević et al. (2002), contains tables where measured values are listed and compared with semi-classical calculations. The Bibliography of Line Shapes and Shifts contains more recent data, see section 3 on Databases. There are also several papers on Stark broadening and plasmainduced line shifts among the articles published as a tribute to Charles Hooper under the collective title Radiative Properties of Hot Dense Matter (Lee 2003). An overview of the role of the plasma micro field in the Stark broadening of spectral lines has been presented by Demura (2004) and Demura and Gilles (2004). These articles contain many useful references.

A short review of the French-Serbian collaboration on theoretical calculations of Stark broadening of spectral line shapes for non-hydrogenic atoms within the framework of the semi-classical approach has been presented by Dimitrijević and Sahal-Bréchot (2004).

\section{Developments in line broadening theory}

Alexiou and Poquérusse (2005) and Poquérusse and Alexiou (2005) have extended standard semi-classical impact theory for hydrogen and hydrogenic ions to include penetrating collisions. Alexiou and Lee (2005) have tested this theory against full quantum mechanical calculations for hydrogen and have found that the difference between the two is much reduced. Bouguettaia et al. (2005) have re-derived results for Lyman- $\alpha$ using a path integral formalism that treats the electron and proton perturbers on the same basis.

Elabidi et al. (2004b) have obtained a new quantum-mechanical formula for the electron impact width and shift of isolated lines using intermediate coupling and a quantum kinetic theory approach to the line broadening of isolated lines with stationary nonequilibrium level populations has been developed by Iglesias (2005).

\section{Isolated lines}

The term 'isolated lines' means lines between atomic levels that are sufficiently separated from the other atomic levels to enable the Stark effect to be treated within perturbation theory (second-order Stark effect). The line broadening and shift is dominated in this case by the interaction with the plasma electrons. Recent theoretical results have been obtained for lines of Ag I (Dimitrijević and Sahal-Bréchot 2003), Be III (Dimitrijević et al. 2003), O II and C II (Mahmoudi et al. 2004a, 2004b, 2005), Tl II (Milovanović 2005) and $\mathrm{Cu}$ III and Zn III (Simić et al. 2005). 
Stark broadening of lines of He I, Mg I and Ar I for a perturber density of $10^{16} \mathrm{~cm}^{-3}$ and for temperatures between $2,500 \mathrm{~K}$ and 50,000 $\mathrm{K}$ have been investigated by Zmerli et al. (2005). Theoretical results have also been obtained for lines of Ne I (Milosavljević and Djeniže 2002b), (Milosavljević et al. 2004), Ar I (Valognes et al. 2004, 2005) and Kr I (Milosavljević et al. 2003), (Milosavljević 2005). Calculations of line widths for Ar I (Ben Nessib and Sahal-Bréchot 2004) are compared with the experimental values of Milosavljević and Djeniže (2003a, 2003b, 2003c) and Nikolić et al. (2004). Cadwell and Hüwel (2004) have determined Stark-broadening parameters for 12 Ar I lines from time-resolved emission spectra with an estimated accuracy of $20 \%$.

Stark broadening parameters for Cd I have been calculated by (Simić 2005) for 11 singlets and 13 triplets in the ultraviolet and visible spectral region and 24 triplets in the infrared, for temperatures between $2,500 \mathrm{~K}$ and $50,000 \mathrm{~K}$, and for a perturber density of $10^{16} \mathrm{~cm}^{-3}$. Moreover line widths have been obtained within the modified semi-empirical approach for $10 \mathrm{~F}$ III multiplets, for temperatures $10,000 \mathrm{~K}-300,000 \mathrm{~K}$ and for a perturber density of $10^{17} \mathrm{~cm}^{-3}$. Dimitrijević et al. (2005) have used the impact approximation to calculate line widths and shifts for the $4 \mathrm{p}^{7} \mathrm{P}^{0}-4 \mathrm{~d}^{7} \mathrm{D}$ transitions in Cr I broadened by electrons, protons and ionized helium.

Most of these results have been obtained by using semi-classical theory which provides a powerful tool for the rapid calculation of line widths and shifts.

Quantum-mechanical Stark broadening calculations for lines of Ne VII and Ne VIII which are in intermediate coupling have been carried out by Elabidi et al. (2004a, 2005). Hydrogen-like and helium-like ions of $\mathrm{C}, \mathrm{Si}$ and Ar have been studied by Stambulchik and Maron (2005) using a numerical simulation of the plasma particle motion and find that ion dynamics is very important for these cases.

\section{Hydrogen lines}

The occupation probability method (OPM) is normally applied to the matching of discrete and continuous radiative spectra in dense hydrogenic plasmas (Vitel et al. 2004). An alternative method that removes the matching problem has been developed by D'yachkov (2004) and the results obtained are in good agreement with experimental data.

The Stark broadening of the Radio Recombination Lines (RRLs) of Hydrogen observed from galactic H II regions is controlled by inelastic collisions with charged particles. Gavrilenko and Oks (2004) have presented a method of calculating both the cross sections of such collisions and the Stark broadening of RRLs based on the classical-trajectory Monte Carlo approach.

For lines of hydrogen in the visible and ultraviolet, measurements of widths and shifts for the Balmer $\alpha$ and $\beta$ lines in a laser-induced plasma have been made by Parigger et al. $(2003,2004)$. Stark widths and shifts of the $\mathrm{H} \alpha$ line measured in a flash tube plasma have been compared with theoretical results by Griem and Halenka (2004) and Flih et al. (2003, 2004). Also a theoretical study of the Lyman $\gamma$ line profile of atomic hydrogen perturbed by collisions with protons is reported by Allard et al. (2004).

Spectroscopic diagnostics of warm dense matter are presented by Astapenko et al. (2004).

\section{Neutral Helium lines}

Stark broadening calculations for the spectral line of neutral helium at $6678 \AA$ formed in a non-ideal plasma have been performed by Ben Chaoucha et al. (2004). Stark line profiles for He I lines emitted by dense plasmas have also been calculated by Gigosos et al. (2004) and Ozmar et al. (2004) and compared to the experimental results of Milosavljević and 
Djeniže (2003d). Calculations have also been carried out for lines of He I by Milosavljević and Djeniže (2002a), Li and You (2002) and Bardet et al. (2003).

\subsection{Broadening by neutral atoms and molecules}

A collection of papers concerning the status of the molecular spectroscopic database, HITRAN 2000, has been published by Rothman et al. (2003) and this has recently been updated for the current version HITRAN 2004 by Rothman et al. (2005). These reviews contain papers specific to line broadening and shift phenomena in molecules.

An overview of state-of-the-art simulations of cool stellar atmospheres using the code PHOENIX was presented by Hauschildt and Baron (2005) and Homeier (2005) at the SCSLSA meeting. Molecules are the dominant opacity source and the resonance lines of $\mathrm{Na}$ and $\mathrm{K}$ have line wings that are particularly strong features of these spectra. The main difficulty is the lack of much realistic experimental or theoretical data for collision broadening by the dominant perturbers in these atmospheres, $\mathrm{H}_{2}$ and He. The assumption of Van der Waals broadening gives at best only crude estimates for the line broadening parameters.

\section{Developments in line shape theory}

Beaud and Knopp (2003) have developed a method of scaling rotationally inelastic collisions with an effective angular momentum parameter and applying it to collisioninduced intra-molecular rotational energy transfer. Excellent agreement with experiment has been achieved for the $\mathrm{CO}-\mathrm{CO}, \mathrm{N}_{2}-\mathrm{N}_{2}$ and $\mathrm{N}_{2}-\mathrm{He}$ systems. Wang (2004) has produced a refined version of the non-perturbative pressure-broadening theory of Neilson and Gordon and has obtained good agreement for CO lines in the fundamental band broadened by Ar.

\section{Broadening of atomic lines}

Some theoretical work has been published in the period 2002-2005 and the transitions with the perturbing atoms or molecules are listed below.

Li far-wing profile of the $2 \mathrm{~s}-2 \mathrm{p}$ resonance line broadened by He (Zhu et al. 2005). $\mathrm{Na}$ far-wing line profiles for the 3s-3p resonance line broadened by $\mathrm{He}$, Ar and Xe Chung et al. (2002).

$\mathrm{Na}$ and $\mathrm{K}$ full line profiles including far line wings of the resonance lines broadened by $\mathrm{H}$ and $\mathrm{H}_{2}$ (Allard et al. 2003).

$\mathrm{Na}$ and $\mathrm{K}$ far-wing line profiles of the resonance lines broadened by $\mathrm{H}_{2}$ and $\mathrm{He}$ (Burrows and Volobuyev 2003).

Na line broadening and mixing in the $3 \mathrm{p}-3 \mathrm{~d}$ transition broadened by $\mathrm{H}$ (Sanchez-Fortún Stoker and Dickinson (2003).

$\mathrm{Na}$ line widths for the $3 \mathrm{~s}-3 \mathrm{p}$ and $3 \mathrm{~d}-4 \mathrm{p}$ transitions broadened by He (Peach et al. 2005).

\section{Broadening and shift of molecular lines}

Much more experimental data has been published since the last report was prepared. The molecules are listed below with their perturbing atomic or molecular species.

$\mathrm{D}_{2}$ collision-induced absorption in the fundamental band in $\mathrm{D}_{2}-\mathrm{N}_{2}$ and $\mathrm{D}_{2}-\mathrm{O}_{2}$ mixtures (Varghese et al. 2004).

HCN lines in the $2 \nu_{2}$ region broadened and shifted by HCN and air (Devi et al. 2004). $\mathrm{HNO}_{3}$ millimeter-wave transitions broadened by $\mathrm{N}_{2}$ and $\mathrm{O}_{2}$ (Colmont et al. 2003). $\mathrm{H}_{2} \mathrm{O}$ lines in the $26000-13000 \mathrm{~cm}^{-1}$ region broadened by $\mathrm{H}_{2} \mathrm{O}$ and $\mathrm{N}_{2}$ (Coheur et al. 2002). 
$\mathrm{H}_{2} \mathrm{O} 183 \mathrm{GHz}$ line broadened and shifted by $\mathrm{N}_{2}, \mathrm{O}_{2}$ and air (Tretyakov 2003).

$\mathrm{H}_{2} \mathrm{O}$ self-broadened pure rotational lines (Podobedov et al. 2004).

$\mathrm{H}_{2} \mathrm{O}$ lines in the $\nu_{2}$ band broadened by air (Wagner et al. 2005).

$\mathrm{H}_{2} \mathrm{O}$ lines in the $1.39 \mu$ m region broadened by $\mathrm{N}_{2}, \mathrm{O}_{2}$ and air (Durry et al. 2005).

$\mathrm{H}_{2} \mathrm{~S}$ lines in the $\nu_{2}$ band broadened and shifted by $\mathrm{H}_{2}, \mathrm{D}_{2}, \mathrm{~N}_{2}, \mathrm{O}_{2}$ and $\mathrm{CO}_{2}$ (Kissel et al. 2002).

$\mathrm{HCl} J=1-0$ rotational transition broadened and shifted by $\mathrm{N}_{2}, \mathrm{O}_{2}$ and $\mathrm{Ar}$ (Morino and Yamada 2005).

$\mathrm{CH}_{4}$ two lines of the $2 \nu_{3}$ band broadened by $\mathrm{N}_{2}, \mathrm{O}_{2}$, He and $\mathrm{Ar}$ (Dufour et al. 2003).

$\mathrm{CO} J=5-4$ transition at $576 \mathrm{GHz}$ broadened and shifted by $\mathrm{CO}, \mathrm{N}_{2}$ and $\mathrm{O}_{2}$ (Markov et al. 2002) and by He, Ne, Ar, Kr and Xe (Yamada and Abe 2003).

$\mathrm{CO} R(7)$ and $R(7)$ transitions in the fundamental band broadened by Ar (Mantz et al. 2003).

$\mathrm{CO}$ second overtone band broadened and shifted by $\mathrm{CO}$ and air (Sung and Varanasi 2004).

$\mathrm{CO}_{2}$ lines in the $1.6 \mu \mathrm{m}$ region broadened by $\mathrm{N}_{2}$ and $\mathrm{O}_{2}$ (Pouchet et al. 2004).

$\mathrm{CO}_{2}$ and OCO self-broadened lines near 2.04mm (Le Barbu et al. 2005).

$\mathrm{CF}_{4}$ lines in the $\nu_{4}$ band broadened by He, $\mathrm{N}_{2}$ and $\mathrm{Ar}$ (Domanskaya et al. 2004).

$\mathrm{CF}_{3} \mathrm{CFH}_{2}$ rotational lines broadened by $\mathrm{CF}_{3} \mathrm{CFH}_{2}, \mathrm{~N}_{2}$ and $\mathrm{O}_{2}$ (Cazzoli et al. 2004).

$\mathrm{NH}_{3} 134$ rovibrational transitions in the $\nu_{4}$ and $2 \nu_{2}$ bands broadened by $\mathrm{H}_{2}$ and $\mathrm{N}_{2}$ (Nouri et al. 2004).

$\mathrm{N}_{2} \mathrm{O}$ millimeter lines broadened by $\mathrm{N}_{2}$ and $\mathrm{O}_{2}$ (Rohart et al. 2003).

$\mathrm{O}_{2} 60-\mathrm{GHz}$ band and single line at $118.75 \mathrm{GHz}$ broadened by $\mathrm{N}_{2}, \mathrm{O}_{2}, \mathrm{He}, \mathrm{Ne}, \mathrm{Ar}, \mathrm{H}_{2} \mathrm{O}$, $\mathrm{CO}$ and $\mathrm{CO}_{2}$ (Golubiatnikov et al. 2003).

$\mathrm{O}_{2}$ single line at $118.75 \mathrm{GHz}$ broadened and shifted by $\mathrm{O}_{2}$ and air (Tretyakov et al. 2004).

$\mathrm{O}_{2} A$-band broadened by $\mathrm{He}, \mathrm{Ne}, \mathrm{Ar}, \mathrm{Kr}$ and Xe (Pope et al. 2004).

$\mathrm{O}_{2}-\mathrm{O}_{2}$ collision-induced resonances at $477 \mathrm{~nm}$ and $577 \mathrm{~nm}$ (Sneep et al. 2005).

$\mathrm{O}_{3}$ submillimeter-wave lines broadened by $\mathrm{N}_{2}, \mathrm{O}_{2}$ and air (Yamada and Amano 2005).

$\mathrm{O}_{3}$ millimeter-wave lines broadened by $\mathrm{N}_{2}, \mathrm{O}_{2}$ and air (Colmont et al. 2005).

$\mathrm{PH}_{3} \nu_{2}$ and $\nu_{4}$ bands broadened by $\mathrm{H}_{2}$, He and Ar (Bouanich et al. 2004), (Salem et al. 2004, 2005).

Theoretical results obtained during the period 2002-2005 are listed below.

$\mathrm{H}_{2} \mathrm{O}$ effects of vibration on lines broadened and shifted by $\mathrm{N}_{2}$ (Gamache and Hartmann 2004).

$\mathrm{H}_{2} \mathrm{O} 11$ vibrational bands in the range $3.2-17.76 \mu \mathrm{m}$ broadened by $\mathrm{N}_{2}, \mathrm{O}_{2}$ and air (Gamache 2005).

$\mathrm{CH}_{3} \mathrm{D}$ lines in the $\nu_{2}$ band broadened by He (Féjard et al. 2003).

$\mathrm{CO}$ lines in the fundamental and first two overtone bands broadened and shifted by $\mathrm{H}_{2}$ (Bouanich and Predoi-Cross 2005).

\subsection{Databases}

A 'virtual observatory' has been developed for astronomers to give immediate access to data archives, databases, reference surveys and information services anywhere in the world, see:

$$
\text { cdsarc.u-strasbg.fr, }
$$

and the latest version of the database High resolution Transmission, HITRAN 2004, can be found at:

$$
\text { http://www.hitran.com. }
$$


The current version of the database Gestion et Etude des Informations Spectroscopiques Atmosphériques (GEISA-03) is at:

$$
\text { http://ara.lmd.polytechnique.fr, }
$$

and the Spherical Top data System (STDS), for spherical top molecules is at:

http://www.u-bourgogne.fr/LPUB/sTDS.html.

The National Institute for Standards and Technology (NIST) maintains a database at:

$$
\text { http://www.physics.nist.gov/PhysRefData, }
$$

which contains the Bibliography on Atomic Line Shapes and Shifts up to 1999 and the database at the Observatoire de Paris,

$$
\text { http://amrel.obspm.fr/balss, }
$$

contains the Bibliography up to 2003. The Vienna Atomic Line Database (VALD) can be found at:

$$
\text { http://www.astro.univie.ac.at/ vald, }
$$

and the Belgrade database at:

$$
\text { http://www.aob.bg.yu/BELDATA. }
$$

\author{
N.F. Allard and G. Peach \\ Chairpersons of the Working Group
}

\title{
References
}

Alexiou S. and Lee, R.W., 2005, J. Quant. Spectrosc. Radiat. Transfer, in press.

Alexiou S. and Poquérusse, A., 2005, Phys. Rev. E, 72, 046404.

Allard, N.F., Allard, F., Hauschildt, P.H., Kielkopf, J.F. and Machin, L., 2003, A\&A, 411, L473.

Allard N.F., Kielkopf J.F., Hébrard G. and Peek J. 2004, Eur. Phys. J. D, 29, 7.

Astapenko, V., Bureyeva, L., and Lisitsa, V., 2004, in Spectral Line Shapes, Proceedings of the 17th ICSLS, ed. E. Dalimier, Editions Frontier Group, Paris, p 129.

Bardet, J.P., Valognes, J.C. and Vitel, Y., 2003, J. Quant. Spectrosc. Radiat. Transfer, 76,45 .

Beaud, P. and Knopp, G., 2003, Chem. Phys. Lett., 371, 194.

Ben Chaouacha, H. and Ben Nessib, N. and Sahal-Bréchot, S, 2004, A\&A, 419, 771.

Ben Nessib, N. and Sahal-Bréchot, S., 2004, in Spectral Line Shapes, Proceedings of the 17th ICSLS, ed. E. Dalimier, Editions Frontier Group, Paris, p 385.

Bouanich, J.-P. and Predoi-Cross, A., 2005, J. Mol. Struct. 742, 183.

Bouanich, J.-P., Salem, J., Aroui, H., Walrand, J. and Blanquet, G., 2004, J. Quant. Spectrosc. Radiat. Transfer, 84, 195.

Bouguettaia, H., Chihi, Is., Chenini, K., Meftah, M.T., Khelfaoui, F. and Stamm, R., 2005, J. Quant. Spectrosc. Radiat. Transfer, 94, 335.

Burrows, A. and Volobuyev, M., 2003, ApJ, 583, 985.

Cadwell, L. and Hüwel, L., 2004, J. Quant. Spectrosc. Radiat. Transfer, 83, 579.

Cazzoli, G., Puzzarini, C. and Dore, L., 2004, J. Quant. Spectrosc. Radiat. Transfer, 83,699 .

Chung, H.-K., Shurgalin, M. and Babb, J.F., 2002, in Spectral Line Shapes, vol 12, ed. C.A. Back, 211. 
Coheur, P.-F., Fally, S., Carleer, M., Clerbaux, C., Colin, R., Jenouvrier, A., Mérienne, M.-F., Hermans, C. and Vandaele, A.C., 2002, J. Quant. Spectrosc. Radiat. Transfer, 74, 493.

Colmont, J.-M., Bakri, B., Rohart, F. and Włodarczak, G., 2003, J. Mol. Spectrosc., 220,52 .

Colmont, J.-M., Bakri, B., Rohart, F., Włodarczak, G., Demaison, J., Cazzoli, G., Dore, L. and Puzzarini, C., 2005, J. Mol. Spectrosc., 231, 171.

Demura, A.V., 2004, in Encyclopedia of Low Temperature Plasmas, volume II, Thermodynamics of Plasmas, ed. I. Iosilevski, and A. Starostin, Fizmatlit (in Russian).

Demura, A.V. and Gilles, D., 2004, in Spectral Line Shapes, Proceedings of the 17th ICSLS, ed. E. Dalimier, Editions Frontier Group, Paris, p 121.

Devi, V.M., Benner, D.C., Smith, M.A.H., Rinsland, C.P., Sharpe, S.W. and Sams, R.L., 2004, J. Quant. Spectrosc. Radiat. Transfer, 87, 339.

Dimitrijević, M.S., Dačić, M., Cvetković, Z. and Sahal-Bréchot, S., 2003, A\&A, 400, 791.

Dimitrijević, M.S. and Sahal-Bréchot, S., 2003, Atomic Data and Nuclear Data Tables, 85, 269.

Dimitrijević, M.S. and Sahal-Bréchot, S., 2004, in Spectral Line Shapes, Proceedings of the 17th ICSLS, ed. E. Dalimier, Editions Frontier Group, Paris, p 397.

Dimitrijević, M.S., Ryabchikova, T., Popović, L.C, Shulyak, D. and Tsymbal, V., 2005, A\&A, in press.

Domanskaya, A.V., Tonkov, M.V. and Boissoles, J., 2004, J. Quant. Spectrosc. Radiat. Transfer, 86, 425 .

Dufour, G., Hurtmans, D., Henry, A., Valentin, A. and Lepère, M., 2003, J. Mol. Spectrosc., 221, 80.

Durry, G., Zeninari, V., Parvitte, B., Le Barbu, T., Lefevre, F., Ovarlez, J. and Gamache, R.R., 2005, J. Quant. Spectrosc. Radiat. Transfer, 94, 387.

D'yachkov, L., 2004, in Spectral Line Shapes, Proceedings of the 17th ICSLS, ed. E. Dalimier, Editions Frontier Group, Paris, p 113.

Elabidi, H., Ben Nessib, N., Cornille, M., Dubau, J. and Sahal-Bréchot, S., 2004a, in Spectral Line Shapes, Proceedings of the 17th ICSLS, ed. E. Dalimier, Editions Frontier Group, Paris, p 430.

Elabidi, H., Ben Nessib, N. and Sahal-Bréchot, S., 2004b, J. Phys. B: At. Mol. Opt. Phys., 37, 63.

Elabidi, H., Ben Nessib, N. and Dimitrijević, M.S., 2005, Proceedings of the 5th Serbian Conference on Spectral Line Shapes in Astrophysics, Mem. S.A.It. 7, 213.

Féjard, L., Gabard, T. and Champion, J.-P., 2003, J. Mol. Spectrosc., 219, 88.

Flih, S.A., Oks, E. and Vitel, Y., 2003, J. Phys. B: At. Mol. Opt. Phys., 36, 283.

Flih, S.A., Oks, E. and Vitel, Y., 2004, in Spectral Line Shapes, Proceedings of the 17th ICSLS, ed. E. Dalimier, Editions Frontier Group, Paris, p 445.

Gamache, R.R., 2005, J. Mol. Spectrosc., 229, 9.

Gamache, R.R. and Hartmann, J.-M., 2004, J. Quant. Spectrosc. Radiat. Transfer, 83, 119.

Gavrilenko, V.P. and Oks, E., 2004, in Spectral Line Shapes, Proceedings of the 17th ICSLS, ed. E. Dalimier, Editions Frontier Group, Paris, p 105.

Gigosos, M.A., González, M.A., Talin, B. and Calisti, A., 2004, in Spectral Line Shapes, Proceedings of the 17th ICSLS, ed. E. Dalimier, Editions Frontier Group, Paris, p 451.

Golubiatnikov, G.Yu., Koshelev, M.A. and Krupnov, A.F., 2003, J. Mol. Spectrosc., $222,191$. 
Griem, H.R. and Halenka, J., 2004, in Spectral Line Shapes, Proceedings of the 17th ICSLS, ed. E. Dalimier, Editions Frontier Group, Paris, p 442.

Hauschildt, P.H. and Baron, E., 2005, Proceedings of the 5th Serbian Conference on Spectral Line Shapes in Astrophysics, Mem. S.A.It. 7, 137.

Homeier, D., 2005, Proceedings of the 5th Serbian Conference on Spectral Line Shapes in Astrophysics, Mem. S.A.It. 7, 157.

Iglesias, C.A., 2005, High Energy Density Physics, in press.

Kissel, A., Sumpf, B., Kronfeldt, H.-D., Tikhomirov, B.A. and Ponomarev, Yu.N., 2002, J. Mol. Spectrosc., 216, 345.

Konjević, N., Lesage, A., Fuhr, J.R. and Wiese, W.L., 2002, J. Phys. Chem. Ref. Data, 31,819 .

Le Barbu, T., Zéninari, V., Parvitte, B., Courtois, D. and Durry, G., 2005, J. Quant. Spectrosc. Radiat. Transfer, in press.

Lee, R.W. (ed.), 2003, Radiative Properties of Dense Matter, J. Quant. Spectrosc. Radiat. Transfer, 81, 1 (special issue).

Li H. and You J., 2002, Chinese Astron. \& Astrophys., 26, 293.

Mahmoudi, W., Ben Nessib, N. and Dimitrijević, M.S., 2004a, A\&A, 434, 773.

Mahmoudi, W., Ben Nessib, N., Dimitrijević, M.S. and Sahal-Bréchot, S., 2005, Proceedings of the 5th Serbian Conference on Spectral Line Shapes in Astrophysics, Mem. S.A.It. 7, 217.

Mahmoudi, W., Ben Nessib, N. and Sahal-Bréchot, S., 2004b, Phys. Scripta, 70, 142.

Mantz, A.W., Thibault, F., Cacheiro, J.L., Fernandez, B., Pedersen, T.B., Koch, H., Valentin, A., Claveau, C., Henry, A. and Hurtmans, D., 2003, J. Mol. Spectrosc., 222, 131.

Markov, V.N., Golubiatnikov, G.Yu., Savin, V.A., Sergeev, D.A., Guarnieri, A. and Mäder, H., 2002, J. Mol. Spectrosc., 212,1.

Milosavljević, V., 2005, Proceedings of the 5th Serbian Conference on Spectral Line Shapes in Astrophysics, Mem. S.A.It. 7, 191.

Milosavljević, V. and Djeniže, S., 2002a, New Astronomy, 7, 543.

Milosavljević, V. and Djeniže, S., 2002b, Phys. Lett. A, 305, 70.

Milosavljević, V. and Djeniže, S., 2003a, A\&A, 393, 721.

Milosavljević, V. and Djeniže, S., 2003b, A\&A, 398, 1179.

Milosavljević, V. and Djeniže, S., 2003c, A\&A, 405, 397.

Milosavljević, V. and Djeniže, S., 2003d, Eur. Phys. J. D., 23, 385.

Milosavljević, V., Djeniže, S. and Dimitrijević, M.S., 2003, Phys. Rev. E., 68, 016402.

Milosavljević, V., Djeniže, S. and Dimitrijević, M.S., 2004, J. Phys. B: At. Mol. Opt. Phys., 37, 2713.

Milovanović, N., 2005, Proceedings of the 5th Serbian Conference on Spectral Line Shapes in Astrophysics, Mem. S.A.It. 7, 129.

Morino, I. and Yamada, K.M.T., 2005, J. Mol. Spectrosc., 233, 77.

Nikolić, D., Djurović, S., Mijatović, Z., Kobilarov, R., Vujičić, B. and Ćirišan, M., 2004, J. Quant. Spectrosc. Radiat. Transfer, 86, 285.

Nouri, S., Orphal, J., Aroui, H. and Hartmann, J.-M., 2004, J. Mol. Spectrosc., 227, 60 .

Ozmar, B., Wierling, A., Günter, S. and Röpke, G., 2004, in Spectral Line Shapes, Proceedings of the 17th ICSLS, ed. E. Dalimier, Editions Frontier Group, Paris, p 150.

Parigger, C.G., Plemmons, D.H. and Oks, E., 2003, Appl. Opt., 42, 5992.

Parigger, C.G., Spectral Line Shapes, 2004, in Spectral Line Shapes, Proceedings of the 17th ICSLS, ed. E. Dalimier, Editions Frontier Group, Paris, p 97. 
Peach, G., Mullamphy, D.F.T., Venturi, V. and Whittingham, I.B., Proceedings of the 5th Serbian Conference on Spectral Line Shapes in Astrophysics, Mem. S.A.It. 7, 145.

Podobedov, V.B., Plusquellic, D.F. and Fraser, G.T., 2004, J. Quant. Spectrosc. Radiat. Transfer, 87, 377.

Pope, R.S., Wolf, P.J. and Perram, G.P., 2004, J. Mol. Spectrosc., 223, 205.

Poquérusse, A. and Alexiou, S., 2005, J. Quant. Spectrosc. Radiat. Transfer, in press.

Pouchet, I., Zéninari, V., Parvitte, B. and Durry, G., 2004, J. Quant. Spectrosc. Radiat. Transfer, 83, 619 .

Rohart, F., Colmont, J.-M., Włodarczak, G. and Bouanich, J.-P., 2003, J. Mol. Spectrosc., 222, 159.

Rothman, L.S., Chance, K. and Goldman, A. (ed.), 2003, J. Quant. Spectrosc. Radiat. Transfer, 82, 1 (HITRAN special issue).

Rothman, L.S., Jacquemart, D., Barbe, A., Benner, D.C., Birk, B., Brown, L.R., Carleer, M.R. et al., 2005, J. Quant. Spectrosc. Radiat. Transfer, 96, 139.

Salem, J., Bouanich, J.-P., Walrand, J., Aroui, H. and Blanquet, G., 2004, J. Mol. Spectrosc., 228, 23.

Salem, J., Bouanich, J.-P., Walrand, J., Aroui, H. and Blanquet, G., 2005, J. Mol. Spectrosc., 232, 247.

Sanchez-Fortún Stoker, J. and Dickinson, A.S., 2003, J. Phys. B: At. Mol. Opt. Phys., 36, 1309 .

Simić, Z., 2005, Proceedings of the 5th Serbian Conference on Spectral Line Shapes in Astrophysics, Mem. S.A.It. 7, 119.

Simić, Z., Popović, L.C, Dimitrijević, M.S. and Dacić, M.D., 2005, Proceedings of the 5th Serbian Conference on Spectral Line Shapes in Astrophysics, Mem. S.A.It. 7, 237.

Sneep, M., Ityaksov, I., Aben, I., Linnartz, H. and Ubachs, W., 2005, J. Quant. Spectrosc. Radiat. Transfer, in press.

Stambulchik, E. and Maron, Y., 2005, J. Quant. Spectrosc. Radiat. Transfer, in press.

Sung, K. and Varanasi, P., 2004, J. Quant. Spectrosc. Radiat. Transfer, 83, 445.

Tretyakov, M.Yu., Golubiatnikov, G.Yu., Parshin, V.V., Koshelev, M.A., Myasnikova, S.E., Krupnov, A.F. and Rosenkranz, P.W., 2004, J. Mol. Spectrosc., 223, 31.

Tretyakov, M.Yu., Parshin, V.V., Koshelev, M.A., Shanin, V.N., Myasnikova, S.E. and Krupnov, A.F., 2003, J. Mol. Spectrosc., 218, 239.

Valognes, J.C., Bardet, J.P., Flih, S.A. and Vitel, Y., 2004, J. Quant. Spectrosc. Radiat. Transfer, 87, 221.

Valognes, J.C., Bardet, J.P., Vitel, Y. and Flih, S.A., 2005, J. Quant. Spectrosc. Radiat. Transfer, 95, 113.

Varghese, G., Stamp, C. and Paddi Reddy, S.P.S., 2004, J. Quant. Spectrosc. Radiat. Transfer, 87, 387.

Vitel, Y., Gavrilova, T.V., D'yachkov, L.G. and Kurilenkov, Yu.K., 2004, J. Quant. Spectrosc. Radiat. Transfer, 83, 387.

Wagner, G., Birk, M., Gamache, R.R. and Hartmann, J.-M., 2005, J. Quant. Spectrosc. Radiat. Transfer, 92, 211.

Wang, W.F., 2004, J. Quant. Spectrosc. Radiat. Transfer, 86, 437.

Yamada, K.M.T. and Abe, H., 2003, J. Mol. Spectrosc., 217, 87.

Yamada, M.M. and Amano, T., 2005, J. Quant. Spectrosc. Radiat. Transfer, 95, 221.

Zhu, C., Babb, J.F. and Dalgarno, A., 2005, Phys. Rev. A, 71, 052710.

Zmerli, B., Ben Nessib, N. and Dimitrijević, M.S., 2005, Proceedings of the 5th Serbian Conference on Spectral Line Shapes in Astrophysics, Mem. S.A.It. 7, 243. 